January 15, 2008 18:6 WSPC/INSTRUCTION FILE barrett-suli-m3assubmission.final

Mathematical Models and Methods in Applied Sciences

(C) World Scientific Publishing Company

\title{
EXISTENCE OF GLOBAL WEAK SOLUTIONS TO DUMBBELL MODELS FOR DILUTE POLYMERS WITH MICROSCOPIC CUT-OFF
}

\author{
JOHN W. BARRETT \\ Department of Mathematics, Imperial College London \\ London SW' $2 A Z, U K$ \\ j.barrett@imperial.ac.uk \\ ENDRE SÜLI \\ University of Oxford \\ Wolfson Building, Parks Road, Oxford OX1 3QD, UK \\ endre.suli@comlab.ox.ac.uk \\ Received (Day Month Year) \\ Revised (Day Month Year) \\ Communicated by (xxxxxxxxxx)
}

\begin{abstract}
We study the existence of global-in-time weak solutions to a coupled microscopicmacroscopic bead-spring model with microscopic cut-off, which arises from the kinetic theory of dilute solutions of polymeric liquids with noninteracting polymer chains. The model consists of the unsteady incompressible Navier-Stokes equations in a bounded domain $\Omega \subset \mathbb{R}^{d}, d=2$ or 3 , for the velocity and the pressure of the fluid, with an elastic extra-stress tensor as the right-hand side in the momentum equation. The extra-stress tensor stems from the random movement of the polymer chains and is defined through the associated probability density function $\psi$ that satisfies a Fokker-Planck-type parabolic equation, a crucial feature of which is the presence of a center-of-mass diffusion term and a cut-off function $\beta^{L}(\psi)=\min (\psi, L)$ in the drag term, where $L \gg 1$. We establish the existence of global-in-time weak solutions to the model for a general class of spring-force potentials including, in particular, the widely used finitely extensible nonlinear elastic (FENE) potential. A key ingredient of the argument is a special testing procedure in the weak formulation of the Fokker-Planck equation, based on the convex entropy function $s \in \mathbb{R}_{\geq 0} \mapsto \mathcal{F}(s):=s(\ln s-1)+1 \in \mathbb{R}_{\geq 0}$. In the case of a corotational drag term, passage to the limit as $L \rightarrow \infty$ recovers the Navier-Stokes-Fokker-Planck model with centre-of-mass diffusion, without cut-off.
\end{abstract}

Keywords: Polymeric flow models; existence of weak solutions; Navier-Stokes equations; Fokker-Planck equations; finitely extensible nonlinear elastic potential.

AMS Subject Classification: 35Q30, 76A05, 76D03, 82C31, 82D60

\section{Introduction}

This paper is concerned with the question of existence of global weak solutions to a system of nonlinear partial differential equations that arises from the kinetic theory 
of dilute polymer solutions. The solvent is an incompressible, viscous, isothermal Newtonian fluid confined to a bounded open set $\Omega \subset \mathbb{R}^{d}, d=2$ or 3 , with boundary $\partial \Omega$. For the sake of simplicity of presentation, we shall suppose that $\Omega$ has solid boundary $\partial \Omega$; the velocity field $\underset{\sim}{u}$ will then satisfy the no-slip boundary condition $\underset{\sim}{u}=\underset{\sim}{0}$ on $\partial \Omega$. The polymer chains, which are suspended in the solvent, are assumed not to interact with each other. The conservation of momentum and mass equations for the solvent then have the form of the incompressible Navier-Stokes equations in which the elastic extra-stress tensor $\underset{\approx}{\tau}$ (i.e., the polymeric part of the Cauchy stress tensor) appears as a source term:

Given $T \in \mathbb{R}_{>0}$, find $\underset{\sim}{u}:(\underset{\sim}{x}, t) \in \bar{\Omega} \times[0, T] \mapsto \underset{\sim}{u}(\underset{\sim}{x}, t) \in \mathbb{R}^{d}$ and $p:(\underset{\sim}{x}, t) \in$ $\Omega \times(0, T] \mapsto p(x, t) \in \mathbb{R}$ such that

$$
\begin{aligned}
& \frac{\partial \underline{\sim}}{\partial t}+\left(\underset{\sim}{u} \cdot \nabla_{x}\right) \underset{\sim}{u}-\nu \Delta_{x} \underset{\sim}{u}+\underset{\sim}{\nabla_{x}} p=\underset{\sim}{f}+\nabla_{x} \cdot \underset{\sim}{\tau} \quad \text { in } \Omega \times(0, T], \\
& \nabla_{x} \cdot \underset{\sim}{u}=0 \quad \text { in } \Omega \times(0, T], \\
& \underset{\sim}{u}=\underset{\sim}{0} \quad \text { on } \partial \Omega \times(0, T], \\
& \underset{\sim}{u}(\underset{\sim}{x}, 0)=\sim_{\sim}(\underset{\sim}{x}) \quad \forall \underset{\sim}{x} \in \Omega,
\end{aligned}
$$

where $\underset{\sim}{u}$ is the velocity field, $p$ is the pressure of the fluid, $\nu \in \mathbb{R}_{>0}$ is the viscosity of the solvent, and $f$ is the density of body forces acting on the fluid.

In the kinetic models under consideration here the extra-stress tensor $\underset{\approx}{\tau}$ is defined via a weighted average of $\psi$, the probability density function of the (random) conformation vector of the polymer molecules (cf. (1.6) below). The Kolmogorov equation satisfied by $\psi$ is a Fokker-Planck-type second-order parabolic equation whose transport coefficients depend on the velocity field $\underset{\sim}{u}$.

Polymer solutions exhibit a range of non-Newtonian flow properties: in particular, the stress endured by a fluid element depends upon the history of deformations experienced by that element. Thereby, rheological properties of non-Newtonian fluids are governed by the flow-induced evolution of their internal microstructure. Following Keunings ${ }^{20}$, a relevant feature of the microstructure is the conformation of the macromolecules, i.e., their orientation and the degree of stretching they experience. From the macroscopic viewpoint it is only the statistical distribution of conformations that matters: the macroscopic stress carried by each fluid element is governed by the distribution of polymer conformations within that element. Motivated by this observation, kinetic theories of polymeric fluids ignore quantum mechanical and atomistic effects and focus on "coarse-grained" models of the polymeric conformations. Depending on the level of coarse-graining, one may arrive at a hierarchy of kinetic models. For example, a dilute solution of linear polymers in a Newtonian solvent can be described in some detail by the freely jointed bead-rod Kramers chain, which comprises a number of beads (of the order of 100) connected by rigid linear segments. A coarser model of the same polymer is the freely jointed bead-spring chain, a Rouse chain, consisting of a smaller number of beads (of the order of 10) connected linearly by entropic springs. A coarser model still is the 
dumbbell model, which involves two beads connected by a spring; cf. Bird, Curtiss, Armstrong, and Hassager ${ }^{9}$. As has been emphasized by Keunings ${ }^{20}$, such coarsegrained models are not meant to capture the detailed structure of the polymer. Rather, they are intended to describe, in more or less detail, the evolution of polymer conformations in a macroscopic flow.

Many of the interesting properties of dilute polymer solutions can be understood by modelling them as suspensions of simple coarse-grained objects (viz. dumbbells) in a Newtonian fluid. This paper is devoted to the mathematical analysis of dumbbell models that are nonlinearly coupled Navier-Stokes-Fokker-Planck systems of partial differential equations: from the technical viewpoint these relatively simple models already exhibit many of the analytical difficulties encountered in the study of more complex models.

Suppose that the domain of admissible conformations (orientation vectors of polymer chains) $D \subset \mathbb{R}^{d}$ is a balanced convex open set in $\mathbb{R}^{d}$; the term balanced means that $\underset{\sim}{q} \in D$ if, and only if, $\underset{\sim}{q} \in D$. Hence, in particular, $\underset{\sim}{0} \in D$. Typically, $D$ is the whole of $\mathbb{R}^{d}$ or a bounded open $d$-dimensional ball centred at the origin $0 \in \mathbb{R}^{d}$.

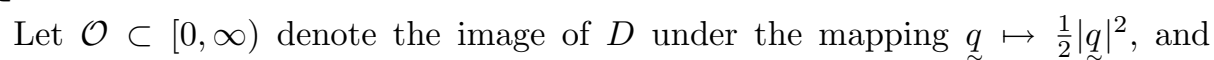
consider the spring-potential $U \in C^{\infty}\left(\mathcal{O} ; \mathbb{R}_{\geq 0}\right)$. Clearly, $0 \in \mathcal{O}$. We shall suppose that $U(0)=0$ and that $U$ is monotonic increasing and unbounded on $\mathcal{O}$. The elastic spring-force $\underset{\sim}{F}: D \subseteq \mathbb{R}^{d} \rightarrow \mathbb{R}^{d}$ is then defined by

$$
\underset{\sim}{F}(\underset{\sim}{q})=U^{\prime}\left(\frac{1}{2}|\underset{\sim}{q}|^{2}\right) \underset{\sim}{q} .
$$

Example 1.1. In the Hookean dumbbell model, the spring force is defined by $\underset{\sim}{F}(\underset{\sim}{q})=$ $\underset{\sim}{q}$, with $\underset{\sim}{q} \in D=\mathbb{R}^{d}$, corresponding to $U(s)=s, s \in \mathcal{O}=[0, \infty)$. Unfortunately, this simple model is physically unrealistic as it admits arbitrarily large extensions. We shall therefore assume in what follows that $D$ is a bounded open ball in $\mathbb{R}^{d}$ centred at the origin $\underset{\sim}{0} \in \mathbb{R}^{d}$. $\diamond$

We shall further suppose that there exist constants $c_{i}>0, i=1,2,3,4$, and $\gamma>1$ such that the (normalized) Maxwellian $M$, defined by

$$
M(\underset{\sim}{q})=\frac{\mathrm{e}^{-U\left(\frac{1}{2}|\underline{\sim}|^{2}\right)}}{\int_{D} \mathrm{e}^{-U\left(\frac{1}{2}|q|^{2}\right)} \mathrm{d} \underset{\sim}{q}},
$$

and the associated potential $U$ satisfy

$$
\begin{gathered}
c_{1}[\operatorname{dist}(\underset{\sim}{q}, \partial D)]^{\gamma} \leq M(\underset{\sim}{q}) \leq c_{2}[\operatorname{dist}(\underset{\sim}{q}, \partial D)]^{\gamma} \quad \forall \underset{\sim}{q} \in D, \\
c_{3} \leq[\operatorname{dist}(\underset{\sim}{q}, \partial D)] U^{\prime}\left(\frac{1}{2}|\underset{\sim}{q}|^{2}\right) \leq c_{4} \quad \forall \underset{\sim}{q} \in D .
\end{gathered}
$$

Observe that

$$
M(\underset{\sim}{q}) \underset{\sim}{\nabla_{q}}[M(\underset{\sim}{q})]^{-1}=-[M(\underset{\sim}{q})]^{-1}{\underset{\sim}{q}}_{q} M(\underset{\sim}{q})=\nabla_{q} U\left(\frac{1}{2}|\underset{\sim}{q}|^{2}\right)=U^{\prime}\left(\frac{1}{2}|\underset{\sim}{q}|^{2}\right) \underset{\sim}{q} .
$$


Since $\left[U\left(\frac{1}{2}|q|^{2}\right)\right]^{2}=(-\ln M(q)+\text { Const. })^{2}$, it follows from (1.3a,b) that (if $\gamma>1$, as has been assumed here, then)

$$
\int_{D}\left[1+\left[U\left(\frac{1}{2}|q|^{2}\right)\right]^{2}+\left[U^{\prime}\left(\frac{1}{2}|q|^{2}\right)\right]^{2}\right] M(\underset{\sim}{q}) \mathrm{d} q<\infty
$$

Example 1.2. In the FENE (finitely extensible nonlinear elastic) dumbbell model the spring force is given by

$$
\underset{\sim}{F}(\underset{\sim}{q})=\frac{1}{1-|q|^{2} / b} \underset{\sim}{q}, \quad \underset{\sim}{q} \in D=B\left(\underset{\sim}{0}, b^{\frac{1}{2}}\right),
$$

corresponding to $U(s)=-\frac{b}{2} \ln \left(1-\frac{2 s}{b}\right), s \in \mathcal{O}=\left[0, \frac{b}{2}\right)$. Here $B\left(0, b^{\frac{1}{2}}\right)$ is a bounded open ball in $\mathbb{R}^{d}$ centred at the origin $\underset{\sim}{0} \in \mathbb{R}^{d}$ and of fixed radius $b^{\frac{1}{2}}$, with $b>0$. Direct calculations show that the Maxwellian $M$ and the elastic potential $U$ of the FENE model satisfy conditions $(1.3 a, b)$ with $\gamma=\frac{b}{2}$ provided that $b>2$. Thereby (1.5) also holds for $b>2$.

It is interesting to note that in the (equivalent) stochastic version of the FENE model a solution to the system of stochastic differential equations associated with the Fokker-Planck equation exists and has trajectorial uniqueness if, and only if, $b>2$ (cf. Jourdain, Lelièvre, and Le Bris ${ }^{18}$ for details). Thus, the assumption $\gamma>1$ can be seen as the weakest reasonable requirement on the decay-rate of $M$ in (1.3a) as $\operatorname{dist}(\underset{\sim}{q}, \partial D) \rightarrow 0 . \quad \diamond$

Due to the flow-induced thermal agitation, polymer molecules are subjected to Brownian forces. Let $(\underset{\sim}{x}, \underset{\sim}{q}, t) \mapsto \psi(\underset{\sim}{x}, \underset{\sim}{q}, t)$ denote the probability density function corresponding to the vector-valued stochastic process $\underset{\sim}{X}(t), Q(t))$, where $\underset{\sim}{X}(t) \in \Omega$ is the position vector of the centre of mass of the dumbbell at time $t \geq 0$, and $Q(t) \in D$ is the conformation (or end-to-end) vector of the dumbbell at time $t \geq 0$. Roughly speaking, $\psi(\underset{\sim}{x}, q, t)$ represents the probability at time $t$ of finding the centre of mass of a dumbbell at $\underset{\sim}{x}$ and having elongation vector $q$.

The governing equations of the coupled Navier-Stokes- Fokker-Planck model are $(1.1 \mathrm{a}-\mathrm{d})$, where the extra-stress tensor $\underset{\approx}{\tau}$ is defined by

$$
\underset{\sim}{\tau}(\underset{\sim}{x}, t)=k \mu\left(\int_{D} \underset{\sim}{q}{\underset{\sim}{q}}^{\top} U^{\prime}\left(\frac{1}{2}|\underset{\sim}{q}|^{2}\right) \psi(\underset{\sim}{x} \underset{\sim}{q}, t) \mathrm{d} \underset{\sim}{q}-\rho(\underset{\sim}{x}, t) \underset{\sim}{I}\right),
$$

with the density of polymer chains located at $\underset{\sim}{x}$ at time $t$ given by

$$
\rho(\underset{\sim}{x}, t)=\int_{D} \psi(\underset{\sim}{x} \underset{\sim}{q}, t) \mathrm{d} q .
$$

The probability density function $\psi$ is a solution of the Fokker-Planck equation

$$
\frac{\partial \psi}{\partial t}+\left(\underset{\sim}{u} \cdot \nabla_{x}\right) \psi+\underset{\sim}{\nabla_{q}} \cdot\left(\underset{\sim}{\sigma}(\underset{\sim}{u} \underset{\sim}{q} \psi)=\frac{1}{2 \lambda} \nabla_{q} \cdot\left(\nabla_{q} \psi+U^{\prime}\left(\frac{1}{2}|\underset{\sim}{\mid q}|^{2}\right) \underset{\sim}{q} \psi\right)+\varepsilon \Delta_{x} \psi,\right.
$$

with $\underset{\sim}{\sigma}(\underset{\sim}{v}) \equiv \underset{\sim}{\nabla_{x}} \underset{\sim}{v}$, where $\left(\underset{\sim}{\nabla_{x}} \underset{\sim}{v}\right)(\underset{\sim}{x}, t) \in \mathbb{R}^{d \times d}$ and $\left\{\underset{\approx}{\nabla_{x}} \underset{\sim}{v}\right\}_{i j}=\frac{\partial v_{i}}{\partial x_{j}}$ (cf. Barrett and Süli $\left.{ }^{5}\right)$. Here, $\varepsilon=\ell_{0}^{2} /(8 \lambda)$ is the centre-of-mass diffusion coefficient of the dumbbells, 
$\ell_{0} \ll \operatorname{diam}(\Omega)$ is the characteristic microscopic length scale (i.e. the characteristic dumbbell size) and $\lambda=\zeta / 4 H$. The parameter $\lambda \in \mathbb{R}_{>0}$ characterizes the elastic relaxation property of the fluid, $\zeta>0$ is a friction coefficient, $H>0$ is a springconstant, $k>0$ is the Boltzmann constant and $\mu>0$ is the absolute temperature.

A noteworthy feature of (1.11) compared to classical Fokker-Planck equations for bead-spring models in the literature is the presence of the $\underset{\sim}{x}$-dissipative centreof-mass diffusion term $\varepsilon \Delta_{x} \psi \equiv\left(\ell_{0}^{2} / 8 \lambda\right) \Delta_{x} \psi$ on the right-hand side of the FokkerPlanck equation (1.8). We refer to Barrett and Süli ${ }^{5}$ for the derivation of (1.8) and the mathematical justification of the presence of the centre-of-mass diffusion term $\varepsilon \Delta_{x} \psi$; see also the recent article by Schieber $^{32}$ concerning generalized dumbbell models with centre-of-mass diffusion. In standard derivations of bead-spring models the centre-of-mass diffusion term is routinely omitted, on the grounds that it is several orders of magnitude smaller than the other terms in the equation. Indeed, when $L \approx 1$ is a characteristic macroscopic length scale (such as, for example, $\operatorname{diam}(\Omega)$ ), Bhave, Armstrong, and Brown ${ }^{8}$ estimate the ratio $\ell_{0}^{2} / L^{2}$ to be in the range of about $10^{-9}$ to $10^{-7}$. However, the omission of the term $\varepsilon \Delta_{x} \psi$ from (1.8) in the case of a heterogeneous solvent velocity $\underset{\sim}{u}(\underset{\sim}{x}, t)$ is a mathematically counterproductive model reduction. When $\varepsilon \Delta_{x} \psi$ is absent, (1.8) becomes a degenerate parabolic equation exhibiting hyperbolic behaviour with respect to $\underset{\sim}{x}, t)$. Since the study of weak solutions to the coupled problem requires one to work with velocity fields $\underset{\sim}{u}$ that have very limited Sobolev regularity (typically $\underset{\sim}{u} \in L^{\infty}\left(0, T ; \underset{\sim}{L^{2}}(\Omega)\right) \cap L^{2}(0, T ; \underset{\sim}{\underset{\sim}{H}}(\Omega))$ ), one is then forced into the technically unpleasant framework of hyperbolically degenerate parabolic equations with rough transport coefficients (cf. Ambrosio ${ }^{1}$ and DiPerna and Lions ${ }^{12}$ ). The resulting difficulties are further exacerbated by the fact that, when $D$ is bounded, a typical spring force $\underset{\sim}{F}(\underset{\sim}{q})$ for a finitely extensible model (such as FENE) explodes as $q$ approaches $\partial D$; see Example 1.2 above. For these reasons, here we shall retain the centre-of-mass diffusion term in (1.8).

We conclude this introduction with a brief survey of recent developments on the analysis of classical bead-spring models; with the exception of Barrett and Süli ${ }^{5}$ mentioned above and El-Kareh and Leal ${ }^{15}$, all articles cited consider models that correspond to formally letting $\varepsilon=0$ in (1.8), i.e., omitting the centre-of-mass diffusion term.

An early contribution to the existence and uniqueness of local-in-time solutions to a family of bead-spring type polymeric flow models is due to Renardy ${ }^{31}$. While the class of potentials $\underset{\sim}{F}(q)$ considered by Renardy ${ }^{31}$ (cf. hypotheses $(\mathrm{F})$ and $\left(\mathrm{F}^{\prime}\right)$ on pp. 314-315) does include the case of Hookean dumbbells, it excludes the practically relevant case of the FENE model (see Example 1.2 above). More recently, E, Li, and Zhang $^{14}$ and Li, Zhang, and Zhang ${ }^{22}$ have revisited the question of local existence of solutions for dumbbell models. A further development in this direction is the work of Zhang and Zhang ${ }^{38}$, where the local existence of regular solutions to FENE-type models has been shown. All of these papers require high regularity of the initial data. 
Constantin ${ }^{10}$ has considered the Navier-Stokes equations coupled to nonlinear Fokker-Planck equations describing the evolution of the probability distribution of the particles interacting with the fluid. He described, in the case when $D$ is a Riemannian manifold, relations determining the coefficients of the stresses added in the fluid by the particles; these relations link the extra stresses to the kinematic effect of the fluid velocity on the particles and to the interparticle interaction potential. In equations (of Type 1 , in the terminology of Constantin ${ }^{10}$ ) where the extra stresses depend linearly on the particle distribution density, as is the case in the present paper, the energy balance requires a response potential. In equations (of Type 2) where the added stresses depend quadratically on the particle distribution, it is shown that energy balance can be achieved without a dynamic response potential, and global existence of smooth solutions is shown if inertial effects are neglected. The necessary relationship (eq. (2.14) in Constantin ${ }^{10}$ ) for the existence of a Lyapunov function in the sense of Theorem 2.2 of Constantin ${ }^{10}$ does not hold for the polymer models considered in the present paper.

Otto and Tzavaras ${ }^{30}$ have investigated the Doi model (which is similar to a Hookean model (cf. Example 1.1 above), except that $D=S^{2}$ ) for suspensions of rod-like molecules in the dilute regime. For certain parameter values, the velocity gradient vs. stress relation defined by the stationary and homogeneous flow is not rank-one monotone. They considered the evolution of possibly large perturbations of stationary flows and proved that, even in the absence of a microscopic cutoff, discontinuities in the velocity gradient cannot occur in finite time.

Jourdain, Lelièvre, and Le Bris ${ }^{18}$ studied the existence of solutions to the FENE model in the case of a simple Couette flow. By using tools from the theory of stochastic differential equations, they established the existence of a unique local-in-time solution to the FENE model in two space dimensions $(d=2)$ when the velocity field $\underset{\sim}{u}$ is unidirectional and of the particular form $\underset{\sim}{u}\left(x_{1}, x_{2}\right)=\left(u_{1}\left(x_{2}\right), 0\right)^{\top}$. The notion of solution for which existence is proved in the paper of Jourdain, Lelièvre, and Le Bris ${ }^{18}$ is mixed deterministic-stochastic in the sense that it is deterministic in the "macroscopic" variable $\underset{\sim}{x}$ but stochastic in the "microscopic" variable $q$. In contrast, our notion of solution (cf. section 3 below) is deterministic both macroscopically and microscopically, since the microscales are modelled here by the probability density function $\psi(\underset{\sim}{x}, \underset{\sim}{q}, t)$. The choice between these different notions of solution has far-reaching consequences on computational simulation: mixed deterministic-stochastic notions of solution necessitate the use of Monte Carlo-type algorithms for the numerical approximation of polymer configurations, as proposed in the monograph of Öttinger ${ }^{29}$ and, for example, in the paper of Jourdain, Lelièvre, and Le Bris ${ }^{17}$; whereas weak solutions in the sense considered in the present paper can be approximated by entirely deterministic (e.g., Galerkin-type) schemes, as was done, for example, in Lozinski, Chauvière, Fang, and Owens ${ }^{26}$ and Lozinski, Owens, and Fang 27 - at the cost of solving a Fokker-Planck equation in $2 d$ spatial dimensions. 
In the case of Hookean dumbbells, and assuming $\varepsilon=0$, the coupled microscopicmacroscopic model described above yields, formally, taking the second moment of $\underset{\sim}{q} \mapsto \psi(\underset{\sim}{q}, \underset{\sim}{x}, t)$, the fully macroscopic, Oldroyd-B model of viscoelastic flow. Lions and Masmoudi ${ }^{24}$ have shown the existence of global-in-time weak solutions to the Oldroyd-B model in a simplified corotational setting (i.e. with $\sigma(\underset{\sim}{u})=\underset{\sim}{\nabla_{x}} \underset{\sim}{u}$ replaced by $\left.\frac{1}{2}\left({\underset{\sim}{\nabla}}_{x} \underset{\sim}{u}-\left({\underset{\sim}{\nabla}}_{x} u\right)^{\top}\right)\right)$. The argument of Lions and Masmoudi ${ }^{24}$ is based on exploiting the propagation in time of the compactness of the solution (i.e. the property that if one takes a sequence of weak solutions which converges weakly and such that the corresponding sequence of initial data converges strongly, then the weak limit is also a solution) and the DiPerna-Lions ${ }^{12}$ theory of renormalized solutions to linear hyperbolic equations with nonsmooth transport coefficients. It is not known if an identical global existence result for the Oldroyd-B model also holds in the absence of the crucial assumption that the drag term is corotational. We note in passing that, assuming $\varepsilon>0$, the coupled microscopic-macroscopic model above yields, taking the appropriate moments in the case of Hookean dumbbells, a dissipative version of the Oldroyd-B model. In this sense, the Hookean dumbbell model has a macroscopic closure: it is the Oldroyd-B model when $\varepsilon=0$, and a dissipative version of Oldroyd-B when $\varepsilon>0$ (cf. Barrett and Süli ${ }^{5}$ ). In contrast, the FENE model is not known to have an exact closure at the macroscopic level, though $\mathrm{Du}, \mathrm{Yu}$, and $\mathrm{Liu}^{13}$ and $\mathrm{Yu}, \mathrm{Du}$, and $\mathrm{Liu}^{37}$ have recently considered the analysis of approximate closures of the FENE model. Lions and Masmoudi ${ }^{25}$ proved the global existence of weak solutions for the corotational FENE dumbbell model, once again corresponding to the case of $\varepsilon=0$, and the Doi model, also called the rod model. As in Lions and Masmoudi ${ }^{24}$, the proof is based on propagation of compactness; see also the related preprint of Masmoudi ${ }^{28}$.

Previously, El-Kareh and Leal ${ }^{15}$ had proposed a macroscopic model, with added dissipation in the equation that governs the evolution of the conformation tensor $\underset{\approx}{A}(\underset{\sim}{x}, t):=\int_{D} \underset{\sim}{q} \underset{\sim}{q}{ }^{\top} U^{\prime}\left(\frac{1}{2}|\underset{\sim}{q}|^{2}\right) \psi(\underset{\sim}{x}, \underset{\sim}{q}, t) \mathrm{d} q \underset{\sim}{q}$, in order to account for Brownian motion across streamlines; the model can be thought of as an approximate macroscopic closure of a FENE-type micro-macro model with centre-of-mass diffusion.

Barrett, Schwab, and $\mathrm{Süli}^{4}$ established the existence of, global in time, weak solutions to the coupled microscopic-macroscopic model (1.1a-d) and (1.8) with $\varepsilon=0$, an $\underset{\sim}{x}$-mollified velocity gradient in the Fokker-Planck equation and an $\underset{\sim}{x}$ mollified probability density function $\psi$ in the Kramers expression-admitting a large class of potentials $U$ (including the Hookean dumbbell model as well as general FENE-type models); in addition to these mollifications, $\underset{\sim}{u}$ in the $\underset{\sim}{x}$-convective term $\left(\underset{\sim}{u} \cdot \nabla_{x}\right) \psi$ in the Fokker-Planck equation was also mollified. Unlike Lions and Masmoudi $^{24}$, the arguments in Barrett, Schwab, and Süli ${ }^{4}$ did not require the assumption that the drag term was corotational in the FENE case. The mollification $S_{\alpha}$ of the velocity field $\underset{\sim}{u}$ that was considered in Barrett, Schwab and $\mathrm{Süli}^{4}$ was stimulated by the Leray- $\alpha$ model of the incompressible Navier-Stokes equations (the viscous Camassa-Holm equations), proposed by Foias, Holm, and Titi ${ }^{16}$, with 
the mollified velocity field $S_{\alpha} \underset{\sim}{u}$ defined as the solution of a Helmholtz-Stokes problem, thus ensuring that the mollified velocity field $S_{\alpha} \underset{\sim}{u}$ is still divergence-free and satisfies the same boundary condition as $\underset{\sim}{u}$.

In Barrett and Süli ${ }^{5}$, we derived the coupled Navier-Stokes-Fokker-Planck model with centre-of-mass diffusion stated above. The anisotropic Friedrichs mollifiers, which naturally arise in the derivation of the model in the Kramers expression for the extra stress tensor and in the drag term in the Fokker-Planck equation, were replaced by isotropic Friedrichs mollifiers. We established the existence of globalin-time weak solutions to the model for a general class of spring-force-potentials including in particular the FENE potential. We justified also, through a rigorous limiting process, certain classical reductions of this model appearing in the literature that exclude the centre-of-mass diffusion term from the Fokker-Planck equation on the grounds that the diffusion coefficient is small relative to other coefficients featuring in the equation. In the case of a corotational drag term we performed a rigorous passage to the limit as the Helmholtz-Stokes mollifiers in the Kramers expression and the drag term converge to identity operators.

In the present paper neither the probability density function $\psi$ in the Kramers expression (1.6) nor the velocity field $\underset{\sim}{u}$ in the drag term

$$
\nabla_{q} \cdot(\underset{\sim}{\sigma}(\underset{\sim}{u}) \underset{\sim}{q} \psi)=\nabla_{q} \cdot\left[\underset{\sim}{\sigma}(\underset{\sim}{u}) \underset{\sim}{q} M\left(\frac{\psi}{M}\right)\right]
$$

appearing in (1.8) will be mollified. Instead, motivated by recent papers of Jourdain, Lelièvre, Le Bris, and Otto ${ }^{19}$ and Lin, Liu, and Zhang ${ }^{23}$ (see also Arnold, Markowich, Toscani, and Unterreiter ${ }^{3}$, and Desvillettes and Villani ${ }^{11}$ ) concerning the convergence of the probability density function $\psi$ to its equilibrium value $\psi_{\infty}(\underset{\sim}{x}, q):=M(q)$ (corresponding to the equilibrium value ${\underset{\sim}{\infty}}_{\infty}(\underset{\sim}{x}):=0 \underset{\sim}{0}$ of the velocity field $\widetilde{\mathrm{d}}$ ) in the absence of body forces $f$, we observe that if $\psi / M$ is bounded above then, for $L \in \mathbb{R}_{>0}$ sufficiently large, the drag term (1.9) is equal to

$$
\nabla_{q} \cdot\left[\underset{\sim}{\sigma}\left(\underset{\sim}{u} \underset{\sim}{q} M \beta^{L}\left(\frac{\psi}{M}\right)\right]\right.
$$

where $\beta^{L} \in C(\mathbb{R})$ is a cut-off function defined as

$$
\beta^{L}(s):= \begin{cases}s & \text { for } s \leq L, \\ L & \text { for } L \leq s .\end{cases}
$$

It follows that, for $L \gg 1$, any solution $\psi$ of (1.8), such that $\psi / M$ is bounded above, also satisfies

$$
\begin{aligned}
\frac{\partial \psi}{\partial t}+\left(\underset{\sim}{u} \cdot \nabla_{x}\right) \psi+\nabla_{q} \cdot\left[\underset{\sim}{\sigma}(\underset{\sim}{u}) \underset{\sim}{q} M \beta^{L}\left(\frac{\psi}{M}\right)\right] \\
=\frac{1}{2 \lambda} \nabla_{q} \cdot\left(M \underset{\sim}{\nabla_{q}}\left(\frac{\psi}{M}\right)\right)+\varepsilon \Delta_{x} \psi \quad \text { in } \Omega \times D \times(0, T] .
\end{aligned}
$$


We impose the following boundary and initial conditions:

$$
\begin{aligned}
& M\left[\frac{1}{2 \lambda} \underset{\sim}{\nabla_{q}}\left(\frac{\psi}{M}\right)-\underset{\sim}{\sigma(u)} \underset{\sim}{q} \beta^{L}\left(\frac{\psi}{M}\right)\right] \cdot \underset{\sim}{\underset{\sim}{|q|}}=0 \quad \text { on } \Omega \times \partial D \times(0, T], \\
& \varepsilon \underset{\sim}{\nabla_{x}} \psi \cdot \underset{\sim}{n}=0 \quad \text { on } \partial \Omega \times D \times(0, T], \\
& \psi(\underset{\sim}{x}, \underset{\sim}{q}, 0)=\psi_{0}(\underset{\sim}{x, q}) \geq 0 \quad \forall(\underset{\sim}{x}, q) \in \Omega \times D,
\end{aligned}
$$

where $q$ is normal to $\partial D$, as $D$ is a bounded ball centred at the origin, and $\underset{\sim}{n}$ is normal to $\partial \Omega$. Here $\int_{D} \psi_{0}(\underset{\sim}{x}, \underset{\sim}{q}) \mathrm{d} q=1$ for a.e. $\underset{\sim}{x} \in \Omega$.

The coupled problem (1.1a-d), (1.6), (1.7), (1.11), (1.12a-c) will be referred to as a dumbbell model with microscopic cut-off. In order to highlight the dependence on $\varepsilon$ and $L$, in subsequent sections the solution to (1.11), (1.12a-c) will be labelled $\psi_{\varepsilon, L}$. Due to the coupling of (1.11) to (1.1a) through (1.6), the velocity and the pressure will also depend on $\varepsilon$ and $L$ and we shall therefore denote them in subsequent sections by $u_{\varepsilon, L}$ and $p_{\varepsilon, L}$.

A detailed argument for introducing cut-off, albeit of a very different nature, was put forward in El-Kareh and Leal ${ }^{15}$ (cf. $(3.10 \mathrm{a}, \mathrm{b})$ ); the authors used a nonnegative function $q \in D \mapsto g(|q|)$ that is compactly supported in $D$, in both the right-hand side of the momentum equation and in the macroscopic counterpart of the Fokker-Planck equation, in order to truncate the unbounded function $\underset{\sim}{q} \in D \mapsto$ $U^{\prime}\left(\frac{1}{2}|q|^{2}\right)=1 /\left(1-|q|^{2} / b\right),|q|^{2}<b$, to a bounded compactly supported function $\underset{\sim}{q} \in D \mapsto g(|q|) U^{\prime}\left(\left.\frac{1}{2}|q|^{2}\right|^{2}\right)$.

The cut-off $\beta^{L}$ proposed here has several attractive properties. We observe that the couple $\left\{{\underset{\sim}{u}}_{\infty}, \psi_{\infty}\right\}$, defined by ${\underset{\sim}{\infty}}_{\infty}(\underset{\sim}{x}):=\underset{\sim}{0}$ and $\psi_{\infty}(\underset{\sim}{x}, q):=M(q)$, is still an equilibrium solution of $(1.1 \mathrm{a}-\mathrm{d})$ with $\underset{\sim}{f}=\underset{\sim}{(1.6)},(1.7),(1.11),(1.12 \mathrm{a}-\mathrm{c})$ for all $L>0$. Thus, unlike the truncation of the (unbounded) potential proposed in ElKareh and Leal ${ }^{15}$, the introduction of the cut-off function $\beta^{L}$ into the Fokker-Planck equation (1.8) does not alter the equilibrium solution $\left(u_{\infty}, \psi_{\infty}\right)$ of the original Navier-Stokes-Fokker-Planck system. In addition, the boundary conditions for $\psi$ on $\partial \Omega \times D \times(0, T]$ and $\Omega \times \partial D \times(0, T]$ ensure that

$$
\frac{1}{|\Omega|} \int_{\Omega \times D} \psi(\underset{\sim}{x} \underset{\sim}{q}, t) \mathrm{d} \underset{\sim}{q} \mathrm{~d} \underset{\sim}{x}=\frac{1}{|\Omega|} \int_{\Omega \times D} \psi_{0}(\underset{\sim}{x}, \underset{\sim}{q}) \mathrm{d} \underset{\sim}{q} \mathrm{~d} x=1 \quad \forall t \in \mathbb{R}_{\geq 0} .
$$

Our objective is to establish the existence of, global in time, weak solutions to the the dumbbell model with microscopic cut-off. The paper is structured as follows. We begin, in section 2, by stating the weak formulation of the coupled Navier-Stokes-Fokker-Planck system with centre-of-mass diffusion and microscopic cut-off, for the general class of potentials $U$ under consideration. In particular, the FENE model fits into the general setting. In section 3 we embark on the proof of existence of weak solutions to our model. We introduce a family of weighted Sobolev spaces that provide the natural functional-analytic framework for the problem: the weight of the space is the Maxwellian induced by the potential $U$ appearing in the Fokker-Planck equation. Our proof requires a special compact embedding result 
in these Maxwellian-weighted Sobolev spaces, which is proved in the appendix by combining compact embedding theorems by Antoci ${ }^{2}$ and Shakhmurov ${ }^{33}$. The proof of existence of global weak solutions to the coupled Navier-Stokes-Fokker-Planck system $(1.1 \mathrm{a}-\mathrm{d}),(1.6),(1.7),(1.11),(1.12 \mathrm{a}-\mathrm{c})$ then rests on a weak-convergence argument. A key ingredient, resulting in sufficiently strong a-priori bounds, is a special testing procedure based on the convex entropy function

$$
s \in \mathbb{R}_{\geq 0} \mapsto \mathcal{F}(s):=s(\ln s-1)+1 \in \mathbb{R}_{\geq 0}
$$

in the weak formulation of the Fokker-Planck equation. This leads to a fortuitous cancellation of the extra stress term on the right-hand side of the NavierStokes equation with the drag term in the Fokker-Planck equation and results in an $L^{\infty}\left(0, T ; L^{1}(\Omega)\right)$ bound on the relative entropy $\mathcal{E}_{M}(\psi)$ of $\psi$ with respect to the equilibrium solution $\psi_{\infty}=M$, where

$$
\mathcal{E}_{M}(\psi):=\int_{D} \mathcal{F}\left(\frac{\psi}{M}\right) M(\underset{\sim}{q}) \mathrm{d} q .
$$

The choice of the entropy function $\mathcal{F}$ in the present context has been motivated by the papers Arnold, Markowich, Toscani, and Unterreiter ${ }^{3}$, Desvillettes and Villani ${ }^{11}$, Jourdain, Lelièvre, Le Bris, and Otto ${ }^{19}$ and Lin, Liu, and Zhang ${ }^{23}$ cited above. It is important to note that the cut-off function $\beta^{L}$ and the entropy function $\mathcal{F}$ are closely related, viz. $\beta^{L}(s)=\min \left(1 / \mathcal{F}^{\prime \prime}(s), L\right)$, and this connection will play a crucial role in our argument. Due to the fact that $\mathcal{F}^{\prime \prime}(s)$ is unbounded at $s=0$, in section 3 the strictly convex entropy function $\mathcal{F}$ will be replaced by a strictly convex regularization $\mathcal{F}_{\delta}^{L}$ whose second derivative is bounded above by $1 / \delta$ and bounded below by $1 / L, \delta \in(0,1), L>1$; at the same time the cutoff function $\beta^{L}$ will be replaced by a strictly positive cut-off function $\beta_{\delta}^{L}$ defined by $\beta_{\delta}^{L}(s)=1 /\left[\mathcal{F}_{\delta}^{L}\right]^{\prime \prime}(s)$. The existence of global weak solutions to the regularized cut-off problem is shown in section 3.1. In section 3.2 we then pass to the limit $\delta \rightarrow 0_{+}$with the regularization parameter $\delta$, to deduce the existence of a global weak solution to the coupled Navier-Stokes-Fokker-Planck system (1.1a-d), (1.6), (1.7), (1.11), (1.12a-c) with microscopic cut-off. Ideally, one would like to replace $\beta^{L}(s)=\min (s, L)$ by $\beta(s)=s$ in the Fokker-Planck equation. However, our current proof of existence in the general non-corotational case requires the presence of the microscopic cut-off function $\beta^{L}$ on the drag term. Nevertheless, in the case of a corotational drag term at least passage to the limit $L \rightarrow \infty$ recovers the Fokker-Planck equation (1.8), without cut-off (see Remark 3.5).

The convergence analysis of a general class of Galerkin-type approximations to the coupled corotational Navier-Stokes-Fokker-Planck model, which is mentioned above and was formulated in Barrett and Süli $^{5}$, was considered in Barrett and $\mathrm{Süli}^{6}$; for the convergence analysis of finite element approximations to the general non-corotational model with cut-off, considered herein, we refer to our forthcoming paper Barrett and Süli ${ }^{7}$. 


\section{The polymer model}

We term polymer models, under consideration here, microscopic-macroscopic-type models, since the continuum mechanical macroscopic equations of incompressible fluid flow are coupled to a microscopic model: the Fokker-Planck equation describing the statistical properties of particles in the continuum. We first present these equations and collect assumptions on the parameters in the model.

Let $\Omega \subset \mathbb{R}^{d}$ be a bounded open set with a Lipschitz-continuous boundary $\partial \Omega$, and suppose that the set $D$ of admissible elongation vectors $\underset{\sim}{q}$ in $(1.8)$ is a bounded open ball in $\mathbb{R}^{d}, d=2$ or 3 , centred at the origin.

Gathering $(1.1 \mathrm{a}-\mathrm{d}),(1.6)$, and (1.8) together, we then consider the following initial-boundary-value problem dependent on the parameters $\varepsilon \ll 1$ and $L \gg 1$ :

$\left(\mathrm{P}_{\varepsilon, L}\right)$ Find $\underset{\sim}{u_{\varepsilon, L}}:(\underset{\sim}{x}, t) \in \bar{\Omega} \times[0, T] \mapsto \underset{\sim}{u_{\varepsilon, L}}(\underset{\sim}{x}, t) \in \mathbb{R}^{d}$ and $p_{\varepsilon, L}:(\underbrace{x}_{\sim}, t) \in$ $\Omega \times(0, T] \mapsto p_{\varepsilon, L}(\underset{\sim}{x}, t) \in \mathbb{R}$ such that

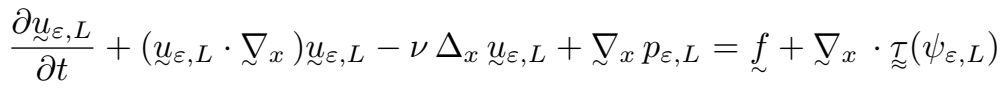

$$
\begin{aligned}
& \text { in } \Omega \times(0, T] \text {, } \\
& \nabla_{x} \cdot{\underset{\sim}{\varepsilon, L}}_{\varepsilon, L}=0 \quad \text { in } \Omega \times(0, T] \\
& {\underset{\sim}{\varepsilon, L}}_{\varepsilon}=\underset{\sim}{0} \quad \text { on } \partial \Omega \times(0, T], \\
& {\underset{\sim}{\varepsilon, L}}_{\varepsilon}(\underset{\sim}{x}, 0)={\underset{\sim}{u}}_{0}(\underset{\sim}{x}) \quad \forall \underset{\sim}{x} \in \Omega,
\end{aligned}
$$

where $\nu \in \mathbb{R}_{>0}$ is the given viscosity, $\underset{\sim}{f}(\underset{\sim}{x}, t)$ is the given body force and $\underset{\sim}{\tau}\left(\psi_{\varepsilon, L}\right)$ : $(\underset{\sim}{x}, t) \in \Omega \times(0, T] \mapsto \underset{\approx}{\tau}\left(\psi_{\varepsilon, L}\right)(\underset{\sim}{x}, t) \in \sim_{\mathbb{R}}^{d \times d}$ is the symmetric extra-stress tensor, dependent on a probability density function $\psi_{\varepsilon, L}:(\underset{\sim}{x} \underset{\sim}{q}, t) \in \bar{\Omega} \times \bar{D} \times[0, T] \mapsto$ $\psi_{\varepsilon, L}(\underset{\sim}{x}, \underset{\sim}{q}, t) \in \mathbb{R}$, defined as

$$
\underset{\approx}{\tau}\left(\psi_{\varepsilon, L}\right)=k \mu\left(\underset{\approx}{C}\left(\psi_{\varepsilon, L}\right)-\rho\left(\psi_{\varepsilon, L}\right) \underset{\approx}{I}\right) .
$$

Here $k, \mu \in \mathbb{R}_{>0}$ are, respectively, the Boltzmann constant and the absolute temperature, $\underset{\approx}{I}$ is the unit $d \times d$ tensor, and

$$
\underset{\approx}{C}\left(\psi_{\varepsilon, L}\right)(\underset{\sim}{x}, t)=\int_{D} \psi_{\varepsilon, L}(\underset{\sim}{x}, \underset{\sim}{q}, t) U^{\prime}\left(\frac{1}{2}|\underset{\sim}{q}|^{2}\right) \underset{\sim}{q} \underset{\sim}{q^{\top}} \mathrm{d} \underset{\sim}{q}
$$

and

$$
\rho\left(\psi_{\varepsilon, L}\right)(\underset{\sim}{x}, t)=\int_{D} \psi_{\varepsilon, L}(\underset{\sim}{x} \underset{\sim}{q}, t) \mathrm{d} \underset{\sim}{q} .
$$

The Fokker-Planck equation with microscopic cut-off satisfied by $\psi_{\varepsilon, L}$ is:

$$
\begin{aligned}
\frac{\partial \psi_{\varepsilon, L}}{\partial t}+ & \left(\underset{\sim}{u_{\varepsilon, L}} \cdot \underset{\sim}{\nabla_{x}}\right) \psi_{\varepsilon, L}+\underset{\sim}{\nabla_{q}} \cdot\left[\underset{\sim}{\sigma}\left(\underset{\sim}{u} \underset{\varepsilon, L}{)} \underset{\sim}{\underset{\sim}{q}} M \beta^{L}\left(\frac{\psi_{\varepsilon, L}}{M}\right)\right]\right. \\
& =\frac{1}{2 \lambda} \nabla_{q} \cdot\left(M \underset{\sim}{\nabla_{q}}\left(\frac{\psi_{\varepsilon, L}}{M}\right)\right)+\varepsilon \Delta_{x} \psi_{\varepsilon, L} \quad \text { in } \Omega \times D \times(0, T] .
\end{aligned}
$$


Here, $\underset{\sim}{\sigma} \underset{\sim}{v}) \equiv \underset{\sim}{\underset{\sim}{\nabla}} \underset{\sim}{v}$ and, for a given $L \gg 1, \beta^{L} \in C(\mathbb{R})$ is defined by (1.10).

We impose the following boundary and initial conditions:

$$
\begin{aligned}
& M\left[\frac{1}{2 \lambda} \underset{\sim}{\nabla_{q}}\left(\frac{\psi_{\varepsilon, L}}{M}\right)-\underset{\sim}{\sigma\left(u_{\varepsilon}, L\right.} \underset{\sim}{q} \beta^{L}\left(\frac{\psi_{\varepsilon, L}}{M}\right)\right] \cdot \underset{\sim}{\frac{q}{|q|}}=0 \quad \text { on } \Omega \times \partial D \times(0, T], \\
& \varepsilon \underset{\sim}{\underset{\sim}{\nabla}} \psi_{\varepsilon, L} \cdot \underset{\sim}{n}=0 \quad \text { on } \partial \Omega \times D \times(0, T], \\
& \psi_{\varepsilon, L}(\underset{\sim}{x, q}, 0)=\psi_{0}(\underset{\sim}{x}, q) \geq 0 \quad \forall(\underset{\sim}{x}, q) \in \Omega \times D
\end{aligned}
$$

where $\underset{\sim}{n}$ is normal to $\partial \Omega$. Here $\int_{D} \psi_{0}(\underset{\sim}{x}, \underset{\sim}{q}) \mathrm{d} q=1$ for a.e. $\underset{\sim}{x} \in \Omega$. The boundary conditions for $\psi_{\varepsilon, L}$ on $\partial \Omega \times D \times(0, T]$ and $\Omega \times \partial D \times(0, T]$ have been chosen so as to ensure that $\int_{\Omega \times D} \psi_{\varepsilon, L}(\underset{\sim}{x} \underset{\sim}{,}, t) \mathrm{d} \underset{\sim}{\mathrm{d}} \underset{\sim}{x}=\int_{\Omega \times D} \psi_{0}(\underset{\sim}{x}, \underset{\sim}{q}) \mathrm{d} \underset{\sim}{q} \mathrm{~d} x=|\Omega|$ for all $t \geq 0$.

\section{Existence of global weak solutions}

Let

$$
\underset{\sim}{H}:=\left\{\underset{\sim}{w} \in \underset{\sim}{L^{2}}(\Omega): \underset{\sim}{\nabla_{x}} \cdot \underset{\sim}{w}=0\right\} \quad \text { and } \quad \underset{\sim}{V}:=\left\{\underset{\sim}{\underset{\sim}{w}} \in \underset{\sim}{H}(\Omega): \underset{\sim}{\nabla}{ }_{x} \cdot \underset{\sim}{w}=0\right\},
$$

where the divergence operator $\nabla_{x}$. is to be understood in the sense of vector-valued

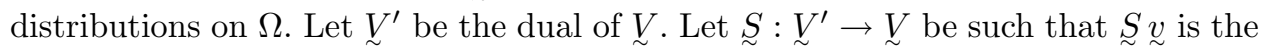
unique solution to the Helmholtz-Stokes problem

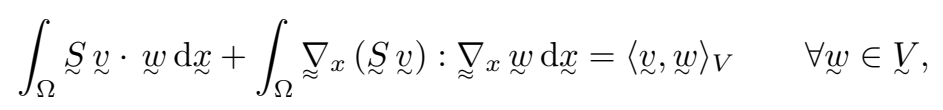

where $\langle\cdot, \cdot\rangle_{V}$ denotes the duality pairing between $\underset{\sim}{V^{\prime}}$ and $\underset{\sim}{V}$. We note that

$$
\langle\underset{\sim}{v}, \underset{\sim}{S} \underset{\sim}{v}\rangle_{V}=\|\underset{\sim}{S} \underset{\sim}{v}\|_{H^{1}(\Omega)}^{2} \quad \forall \underset{\sim}{v} \in V_{\sim}^{\prime} \supset\left(\underset{\sim}{H} H_{0}^{1}(\Omega)\right)^{\prime},
$$

and $\|S \cdot\|_{H^{1}(\Omega)}$ is a norm on $V^{\prime}$.

For later purposes, we recall the following well-known Gagliardo-Nirenberg inequality. Let $r \in[2, \infty)$ if $d=2$, and $r \in[2,6]$ if $d=3$ and $\theta=d\left(\frac{1}{2}-\frac{1}{r}\right)$. Then, there is a constant $C$, depending only on $\Omega, r$ and $d$, such that the following inequality holds for all $\eta \in H^{1}(\Omega)$ :

$$
\|\eta\|_{L^{r}(\Omega)} \leq C\|\eta\|_{L^{2}(\Omega)}^{1-\theta}\|\eta\|_{H^{1}(\Omega)}^{\theta} .
$$

The aim of this paper is to prove existence of a (global-in-time) solution of a weak formulation of the problem $\left(\mathrm{P}_{\varepsilon, L}\right)$ for any fixed parameters $\varepsilon \in(0,1]$ and $L>1$ under the following assumptions on the data:

$$
\begin{aligned}
& \partial \Omega \in C^{0,1}, \quad \underset{\sim}{u_{0} \in \underset{\sim}{H},} \widehat{\psi}_{0}:=M^{-1} \psi_{0} \in L_{M}^{2}(\Omega \times D) \text { with } \widehat{\psi}_{0} \geq 0 \text { a.e. in } \Omega \times D, \\
& \gamma>1 \text { in }(1.3 \mathrm{a}, \mathrm{b}), \quad \text { and } \quad \underset{\sim}{f \in L^{\frac{4}{d}}\left(0, T ; V^{\prime}\right) .}
\end{aligned}
$$


Here $L_{M}^{2}(\Omega \times D)$ is the Maxwellian-weighted $L^{2}$ space over $\Omega \times D$ with norm

$$
\|\widehat{\varphi}\|_{L_{M}^{2}(\Omega \times D)}:=\left\{\int_{\Omega \times D} M|\widehat{\varphi}|^{2} \mathrm{~d} \underset{\sim}{q} \mathrm{~d} x\right\}^{\frac{1}{2}} .
$$

Similarly, we introduce $L_{M}^{2}(D)$, the Maxwellian-weighted $L^{2}$ space over $D$.

On introducing

$$
\|\widehat{\varphi}\|_{H_{M}^{1}(\Omega \times D)}:=\left\{\int_{\Omega \times D} M\left[|\widehat{\varphi}|^{2}+\left|\nabla_{x} \widehat{\varphi}\right|^{2}+\left|\nabla_{q} \widehat{\varphi}\right|^{2}\right] \mathrm{d} \underset{\sim}{q} \mathrm{~d} \underset{\sim}{ }\right\}^{\frac{1}{2}},
$$

we then set

$$
\widehat{X} \equiv H_{M}^{1}(\Omega \times D):=\left\{\widehat{\varphi} \in L_{\mathrm{loc}}^{1}(\Omega \times D):\|\widehat{\varphi}\|_{H_{M}^{1}(\Omega \times D)}<\infty\right\} .
$$

It follows that

$$
C^{\infty}(\overline{\Omega \times D}) \text { is dense in } \widehat{X} \text {. }
$$

This can be shown, for example, by a simple adaptation of Lemma 3.1 in Barrett, Schwab, and Süli ${ }^{4}$, which appeals to fundamental results on weighted Sobolev spaces in Triebe ${ }^{36}$ and Kufner $^{21}$. We have from Sobolev embedding that

$$
L^{s}\left(\Omega ; L_{M}^{2}(D)\right) \hookrightarrow H^{1}\left(\Omega ; L_{M}^{2}(D)\right),
$$

where $s \in[1, \infty)$ if $d=2$ or $s \in[1,6]$ if $d=3$. Similarly to (3.4) we have, with $r$ and $\theta$ as defined there, that there exists a constant $C$, depending only on $\Omega, r$ and $d$, such that

$\|\widehat{\varphi}\|_{L^{r}\left(\Omega ; L_{M}^{2}(D)\right)} \leq C\|\widehat{\varphi}\|_{L^{2}\left(\Omega ; L_{M}^{2}(D)\right)}^{1-\theta}\|\widehat{\varphi}\|_{H^{1}\left(\Omega ; L_{M}^{2}(D)\right)}^{\theta} \quad \forall \widehat{\varphi} \in H^{1}\left(\Omega ; L_{M}^{2}(D)\right)$.

In addition, we note that the embeddings

$$
\begin{aligned}
L_{M}^{2}(D) & \hookrightarrow H_{M}^{1}(D), \\
L_{M}^{2}(\Omega \times D) \equiv L^{2}\left(\Omega ; L_{M}^{2}(D)\right) & \hookrightarrow H_{M}^{1}(\Omega \times D) \equiv L^{2}\left(\Omega ; H_{M}^{1}(D)\right) \cap H^{1}\left(\Omega ; L_{M}^{2}(D)\right)
\end{aligned}
$$

are compact if $\gamma \geq 1$ in $(1.3 \mathrm{a}, \mathrm{b})$; see Appendix A.

Let $\widehat{X}^{\prime}$ be the dual space of $\widehat{X}$ with $L_{M}^{2}(\Omega \times D)$ being the pivot space. Then, similarly to (3.2), let $\mathcal{G}: \widehat{X}^{\prime} \rightarrow \widehat{X}$ be such that $\mathcal{G} \widehat{\eta}$ is the unique solution of

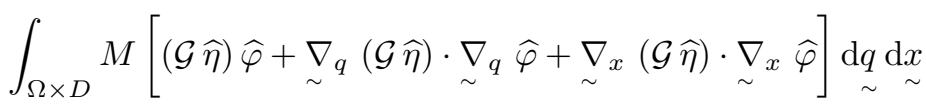

$$
\begin{aligned}
& =\langle M \widehat{\eta}, \widehat{\varphi}\rangle_{\widehat{X}} \quad \forall \widehat{\varphi} \in \widehat{X},
\end{aligned}
$$

where $\langle M \cdot, \cdot\rangle_{\widehat{X}}$ denotes the duality pairing between $\widehat{X}^{\prime}$ and $\widehat{X}$. Then, similarly to (3.3), we have that

$$
\langle M \widehat{\eta}, \mathcal{G} \widehat{\eta}\rangle_{\widehat{X}}=\|\mathcal{G} \widehat{\eta}\|_{\widehat{X}}^{2} \quad \forall \widehat{\eta} \in \widehat{X}^{\prime},
$$

and $\|\mathcal{G} \cdot\|_{\widehat{X}}$ is a norm on $\widehat{X}^{\prime}$. 
We recall the following compactness result, see, e.g., Temam ${ }^{35}$ and Simon ${ }^{34}$. Let $\mathcal{B}_{0}, \mathcal{B}$ and $\mathcal{B}_{1}$ be Banach spaces, $\mathcal{B}_{i}, i=0,1$, reflexive, with a compact embedding $\mathcal{B}_{0} \hookrightarrow \mathcal{B}$ and a continuous embedding $\mathcal{B} \hookrightarrow \mathcal{B}_{1}$. Then, for $\alpha_{i}>1, i=0,1$, the embedding

$$
\left\{\eta \in L^{\alpha_{0}}\left(0, T ; \mathcal{B}_{0}\right): \frac{\partial \eta}{\partial t} \in L^{\alpha_{1}}\left(0, T ; \mathcal{B}_{1}\right)\right\} \hookrightarrow L^{\alpha_{0}}(0, T ; \mathcal{B})
$$

is compact.

Throughout we will assume that (3.5) hold, so that (1.5) and (3.11a,b) hold. We note for future reference that (2.3a) and (1.5) yield that, for $\widehat{\varphi} \in L_{M}^{2}(\Omega \times D)$,

$$
\begin{aligned}
& \int_{\Omega}|C(M \widehat{\sim})|^{2} \mathrm{~d} x=\int_{\Omega} \sum_{i=1}^{d} \sum_{j=1}^{d}\left(\int_{D} M \widehat{\varphi} U^{\prime} q_{i} q_{j} \mathrm{~d} q\right)^{2} \mathrm{~d} x \\
& \leq d\left(\int_{D} M\left(U^{\prime}\right)^{2} \underset{\sim}{|q|^{4}} \mathrm{~d} q\right)\left(\int_{\Omega \times D} M|\widehat{\varphi}|^{2} \underset{\sim}{\mathrm{d} q} \underset{\sim}{\mathrm{d} x}\right) \\
& \leq C\left(\int_{\Omega \times D} M|\widehat{\varphi}|^{2} \underset{\sim}{\mathrm{d} q} \underset{\sim}{\mathrm{d} x}\right),
\end{aligned}
$$

where $C=C(d)$ is a positive constant.

In order to prove existence of weak solutions to $\left(\mathrm{P}_{\varepsilon, L}\right)$, we require a further regularization. Let $\mathcal{F} \in C\left(\mathbb{R}_{>0}\right)$ be defined by

$$
\mathcal{F}(s):=s(\ln s-1)+1, \quad s>0 .
$$

As $\lim _{s \rightarrow 0_{+}} \mathcal{F}(s)=1$, the function $\mathcal{F}$ can be considered to be defined and continuous on $[0, \infty)$, where it is a nonnegative, strictly convex function with $\mathcal{F}(1)=0$.

We then introduce the following convex regularization $\mathcal{F}_{\delta}^{L} \in C^{2,1}(\mathbb{R})$ of $\mathcal{F}$ defined, for any $\delta \in(0,1)$ and $L>1$, by

$$
\mathcal{F}_{\delta}^{L}(s):= \begin{cases}\frac{s^{2}-\delta^{2}}{2 \delta}+s(\ln \delta-1)+1 & \text { for } s \leq \delta, \\ \mathcal{F}(s) \equiv s(\ln s-1)+1 & \text { for } \delta \leq s \leq L, \\ \frac{s^{2}-L^{2}}{2 L}+s(\ln L-1)+1 & \text { for } L \leq s\end{cases}
$$

Hence,

$$
\begin{aligned}
& {\left[\mathcal{F}_{\delta}^{L}\right]^{\prime}(s)= \begin{cases}\frac{s}{\delta}+\ln \delta-1 & \text { for } s \leq \delta, \\
\ln s & \text { for } \delta \leq s \leq L, \\
\frac{s}{L}+\ln L-1 & \text { for } L \leq s,\end{cases} } \\
& {\left[\mathcal{F}_{\delta}^{L}\right]^{\prime \prime}(s)= \begin{cases}\delta^{-1} & \text { for } s \leq \delta, \\
s^{-1} & \text { for } \delta \leq s \leq L, \\
L^{-1} & \text { for } L \leq s .\end{cases} }
\end{aligned}
$$

We note that

$$
\mathcal{F}_{\delta}^{L}(s) \geq \begin{cases}\frac{s^{2}}{2 \delta} & \text { for } s \leq 0 \\ \frac{s^{2}}{4 L}-C(L) & \text { for } s \geq 0\end{cases}
$$


and that $\left[\mathcal{F}_{\delta}^{L}\right]^{\prime \prime}(s)$ is bounded below by $1 / L$ for all $s \in \mathbb{R}$. Finally, we set

$$
\beta_{\delta}^{L}(s):=\left(\left[\mathcal{F}_{\delta}^{L}\right]^{\prime \prime}\right)^{-1}(s)=\max \left\{\beta^{L}(s), \delta\right\},
$$

and observe that $\beta_{\delta}^{L}(s)$ is bounded above by $L$ for all $s \in \mathbb{R}$.

\subsection{Existence for $\left(P_{\varepsilon, L, \delta}\right)$}

$\left(\mathrm{P}_{\varepsilon, L, \delta}\right)$, with solution $\left\{u_{\varepsilon, L, \delta}, \psi_{\varepsilon, L, \delta}\right\}$, will denote problem $\left(\mathrm{P}_{\varepsilon, L}\right)$, where $\beta^{L}(\cdot)$ in (2.4) and (2.5a) is replaced by $\beta_{\delta}^{L}(\cdot)$; recall (1.10) and (3.20). In this subsection we will prove existence of a solution to the following weak formulation of $\left(\mathrm{P}_{\varepsilon, L, \delta}\right)$ for given parameters $\varepsilon, \delta \in(0,1]$ and $L>1$ with $\widehat{\psi}_{\varepsilon, L, \delta}=\psi_{\varepsilon, L, \delta} / M$ :

$\left(\mathrm{P}_{\varepsilon, L, \delta}\right)$ Find $u_{\varepsilon, L, \delta} \in L^{\infty}\left(0, T ; L_{\tilde{\sim}}^{2}(\Omega)\right) \cap L^{2}(0, T ; V) \cap W^{1, \frac{4}{d}}\left(0, T ; V^{\prime}\right)$ and $\widehat{\psi}_{\varepsilon, L, \delta} \in$ $L^{\infty}\left(0, T ; L_{M}^{2}(\Omega \times D)\right) \cap L^{2}(0, T ; \widehat{X}) \cap H^{1}\left(0, T ; \widehat{X}^{\prime}\right)$, with $\underset{\approx}{C}\left(M \widehat{\psi}_{\varepsilon, L, \delta}\right) \in L^{\infty}(0, T ;$ $\left.\underset{\sim}{L^{2}}(\Omega)\right)$, such that ${\underset{\sim}{\varepsilon, L, \delta}}_{\varepsilon}(\cdot, 0)={\underset{\sim}{u}}_{0}(\cdot), \widehat{\psi}_{\varepsilon, L, \delta}(\cdot, \cdot, 0)=\widehat{\psi}_{0}(\cdot, \cdot)$ and

$$
\begin{aligned}
& \int_{0}^{T}\left\langle\frac{\partial u_{\varepsilon, L, \delta}}{\partial t}, \underset{\sim}{w}\right\rangle_{V} \mathrm{~d} t \\
& +\int_{0}^{T} \int_{\Omega}\left[\left[\left(\underset{\sim}{u_{\varepsilon}, L, \delta} \cdot \underset{\sim}{\underset{\sim}{\nabla}}\right) \underset{\sim}{u_{\varepsilon}, L, \delta}\right] \cdot \underset{\sim}{w}+\underset{\sim}{\underset{\sim}{\nabla}} \underset{\sim}{u_{\varepsilon}, L, \delta}: \underset{\sim}{\nabla_{x}} \underset{\sim}{w}\right] \mathrm{d} \underset{\sim}{x} \mathrm{~d} t \\
& =\int_{0}^{T}\langle\underset{\sim}{f f} \underset{\sim}{w}\rangle_{V} \mathrm{~d} t-k \mu \int_{0}^{T} \int_{\Omega} \underset{\sim}{C}\left(M \widehat{\psi}_{\varepsilon, L, \delta}\right): \underset{\approx}{\nabla_{x}} \underset{\sim}{w} \mathrm{~d} x \underset{\sim}{x} \mathrm{~d} t \quad \forall \underset{\sim}{w} \in L^{\frac{4}{4-d}}(0, T ; \underset{\sim}{V}) ; \\
& \int_{0}^{T}\left\langle M \frac{\partial \widehat{\psi}_{\varepsilon, L, \delta}}{\partial t}, \widehat{\varphi}\right\rangle_{\widehat{X}} \mathrm{~d} t \\
& \left.+\int_{0}^{T} \int_{\Omega \times D} M\left[\frac{1}{2 \lambda} \underset{\sim}{\nabla_{q}} \widehat{\psi}_{\varepsilon, L, \delta}-\underset{\sim}{\sigma} \underset{\sim}{\sigma}\left(u_{\varepsilon, L, \delta}\right) \underset{\sim}{q}\right] \beta_{\delta}^{L}\left(\widehat{\psi}_{\varepsilon, L, \delta}\right)\right] \cdot \underset{\sim}{\nabla_{q}} \widehat{\varphi} \underset{\sim}{\mathrm{d} q} \mathrm{~d} x \mathrm{\sim} \mathrm{d} t \\
& +\int_{0}^{T} \int_{\Omega \times D} M\left[\underset{\sim}{\varepsilon} \underset{\sim}{\underset{\psi}{\nabla}} \widehat{\psi}_{\varepsilon, L, \delta}-\underset{\sim}{u_{\varepsilon, L, \delta}} \widehat{\psi}_{\varepsilon, L, \delta}\right] \cdot \underset{\sim}{\nabla_{x}} \widehat{\varphi} \underset{\sim}{\mathrm{d}} \underset{\sim}{\mathrm{d}} x \mathrm{~d} t=0 \quad \forall \widehat{\varphi} \in L^{2}(0, T ; \widehat{X}) .
\end{aligned}
$$

Remark 3.1. If $d=2$, then ${\underset{\sim}{\varepsilon}, L, \delta}_{\varepsilon} \in C([0, T] ; \underset{\sim}{H}$ ) (cf. Lemma 1.2 on p. 176 of Temam ${ }^{35}$ ), whereas if $d=3$, then $u_{\varepsilon, L, \delta}$ is weakly continuous only as a mapping from $[0, T]$ into $\underset{\sim}{H}$ (similarly as in Theorem 3.1 on p. 191 in Temam ${ }^{35}$ ). It is in the latter, weaker sense that the imposition of the initial condition to the $u_{\varepsilon, L, \delta}$-equation will be understood for $d=2,3$ : that is, $\left.\lim _{t \rightarrow 0_{+}} \int_{\Omega}\left({\underset{\sim}{u}, L, \delta}_{\underset{\sim}{*}}(\underset{\sim}{x}, t)-\underset{\sim}{u_{0}}(\underset{\sim}{x})\right) \cdot \underset{\sim}{v} \underset{\sim}{x}\right) \mathrm{d} x=0$ for all $\underset{\sim}{v} \underset{\sim}{H}$. Similarly, for the initial conditions of the $\widehat{\psi}_{\varepsilon, L, \delta}$-equation for $d=2,3$ : $\lim _{t \rightarrow 0_{+}} \int_{\Omega \times D} M\left(\widehat{\psi_{\varepsilon}, L, \delta}(\underset{\sim}{x}, \underset{\sim}{q}, t)-\widehat{\psi}_{0}(\underset{\sim}{x}, \underset{\sim}{q})\right) \widehat{\varphi}(\underset{\sim}{x} \underset{\sim}{q}) \mathrm{d} \underset{\sim}{\mathrm{d}} \mathrm{d} \underset{\sim}{x}=0$ for all $\widehat{\varphi} \in L_{M}^{2}(\Omega \times D)$.

In order to prove existence of a weak solution to $\left(\mathrm{P}_{\varepsilon, L, \delta}\right)$, we discretize in time; and so for any $T>0$, let $N \Delta t=T$ and $t_{n}=n \Delta t, n=0 \rightarrow N$. To prove existence of weak solutions under minimal smoothness requirements on the initial data, recall 
(3.5), we introduce $u_{\sim}^{0} \in \underset{\sim}{V}$ such that

$$
\int_{\Omega}\left[u_{\sim}^{0} \cdot \underset{\sim}{v}+\Delta t \underset{\sim}{\nabla_{x}} \underset{\sim}{u^{0}}: \underset{\sim}{\nabla_{x}} \underset{\sim}{v}\right] \underset{\sim}{\mathrm{d} x}=\int_{\Omega} \underset{\sim}{u_{0}} \underset{\sim}{v} \mathrm{~d} x \quad \underset{\sim}{\forall v} \in \underset{\sim}{V}
$$

and so

$$
\int_{\Omega}\left[\left|u_{\sim}^{0}\right|^{2}+\Delta t\left|\nabla_{x}{\underset{\sim}{u}}^{0}\right|^{2}\right] \mathrm{d} \underset{\sim}{x} \leq \int_{\Omega}\left|u_{0}\right|^{2} \mathrm{~d} x \leq C .
$$

In addition, we have that ${\underset{\sim}{u}}^{0}$ converges to ${\underset{\sim}{0}}_{0}$ weakly in $\underset{\sim}{H}$ in the limit of $\Delta t \rightarrow 0_{+}$.

Let ${\underset{\sim}{\varepsilon, L, \delta}}_{0}^{0}={\underset{\sim}{u}}^{0}$ and $\widehat{\psi}_{\varepsilon, L, \delta}^{0}=\widehat{\psi}_{0}$. Then, for $n=1 \rightarrow N$, given $\left\{u_{\sim, L, \delta}^{n-1}, \widehat{\psi}_{\varepsilon, L, \delta}^{n-1}\right\} \in$ $\underset{\sim}{V} \times L_{M}^{2}(\Omega \times D)$, find $\left\{{\underset{\sim}{u}, L, \delta}_{\varepsilon, \delta}^{n}, \widehat{\psi}_{\varepsilon, L, \delta}^{n}\right\} \in \underset{\sim}{V} \times \widehat{X}$ such that

$$
\begin{aligned}
& \int_{\Omega}\left[\frac{u_{\varepsilon, L, \delta}^{n}-u_{\tau, L, \delta}^{n-1}}{\Delta t}+\left(\underset{\sim}{u_{\varepsilon, L, \delta}^{n-1}} \cdot{\underset{\sim}{\nabla}}_{x}\right) \underset{\sim}{u_{\varepsilon, L, \delta}^{n}}\right] \cdot \underset{\sim}{w} \mathrm{~d} x+\nu \int_{\Omega} \underset{\sim}{\nabla_{x}} \underset{\sim}{u_{\varepsilon, L, \delta}^{n}}: \underset{\sim}{\nabla_{x}} \underset{\sim}{w} \mathrm{~d} x \\
& =\int_{\Omega \sim} f_{\sim}^{n} \cdot \underset{\sim}{w} \mathrm{~d} x-k \mu \int_{\Omega} C\left(M \widehat{\psi}_{\varepsilon, L, \delta}^{n}\right): \underset{\sim}{\nabla} \underset{\sim}{w} \mathrm{~d} x \quad \underset{\sim}{\forall} \underset{\sim}{\underset{\sim}{V}}, \\
& \int_{\Omega \times D} M \frac{\widehat{\psi}_{\varepsilon, L, \delta}^{n}-\widehat{\psi}_{\varepsilon, L, \delta}^{n-1}}{\Delta t} \widehat{\varphi} \mathrm{d} \underset{\sim}{q} \mathrm{~d} x \\
& \left.+\int_{\Omega \times D} M\left[\frac{1}{2 \lambda} \underset{\sim}{\nabla_{q}} \widehat{\psi}_{\varepsilon, L, \delta}^{n}-\underset{\sim}{\sigma} \underset{\sim}{u_{\varepsilon, L, \delta}^{n}} \underset{\sim}{q}\right] \beta_{\delta}^{L}\left(\widehat{\psi}_{\varepsilon, L, \delta}^{n}\right)\right] \cdot \underset{\sim}{\nabla_{q}} \widehat{\varphi} \underset{\sim}{\mathrm{d} q} \mathrm{~d} x \\
& +\int_{\Omega \times D} M\left[\varepsilon{\underset{\sim}{\nabla}}_{x} \widehat{\psi}_{\varepsilon, L, \delta}^{n}-\underset{\sim}{u_{\varepsilon, L, \delta}^{n-1}} \widehat{\psi}_{\varepsilon, L, \delta}^{n}\right] \cdot \underset{\sim}{\nabla_{x}} \widehat{\varphi} \mathrm{d} q \underset{\sim}{\mathrm{d} x}=0 \quad \forall \widehat{\varphi} \in \widehat{X} ;
\end{aligned}
$$

where

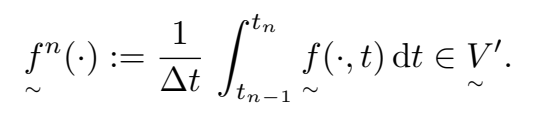

It follows from (3.5) and (3.25) that

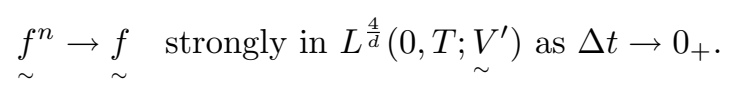

It is convenient to rewrite (3.24a) as

$$
b\left(\sim_{\varepsilon, L, \delta}^{n}, \underset{\sim}{w}\right)=\ell_{b}\left(\widehat{\psi}_{\varepsilon, L, \delta}^{n}\right)(\underset{\sim}{w}) \quad \forall \underset{\sim}{w} \in \underset{\sim}{V}
$$

where for all $\underset{\sim}{w} \in \underset{\sim}{H}{ }_{0}^{1}(\Omega), i=1,2$,

$$
b\left(\underset{\sim}{w_{1}}, \underset{\sim}{w_{2}}\right):=\int_{\Omega}\left[w_{\sim}^{w_{1}}+\Delta t\left(\underset{\sim}{u_{\varepsilon, L, \delta}^{n-1}} \cdot \underset{\sim}{\nabla_{x}}\right) \underset{\sim}{w_{1}}\right] \cdot \underset{\sim}{w_{2}} \mathrm{~d} x+\Delta t \nu \int_{\Omega} \underset{\sim}{\nabla_{x}} \underset{\sim}{w_{1}}: \underset{\sim}{\nabla_{x}} \underset{\sim}{w_{2}} \mathrm{~d} x
$$

and for all $\underset{\sim}{w} \in \underset{\sim}{H}{ }_{0}^{1}(\Omega)$ and $\widehat{\varphi} \in L_{M}^{2}(\Omega \times D)$

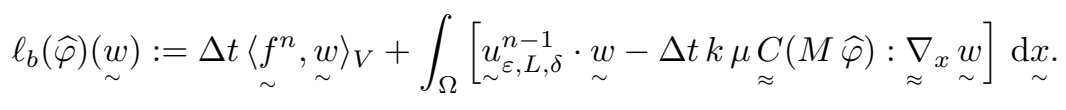


We note that

$$
\begin{aligned}
\int_{\Omega}\left[\left(\underset{\sim}{v} \cdot \nabla_{x}\right) \underset{\sim}{w_{1}}\right] \cdot{\underset{\sim}{w}}_{2} \mathrm{~d} \underset{\sim}{x} \\
\quad=-\int_{\Omega}\left[(\underset{\sim}{v} \cdot \underset{\sim}{\nabla}) \underset{\sim}{w_{2}}\right] \cdot{\underset{\sim}{w}}_{1} \mathrm{~d} \underset{\sim}{x} \quad \forall \underset{\sim}{v} \in V, \quad \forall{\underset{\sim}{1}}_{1},{\underset{\sim}{w}}_{2} \in \underset{\sim}{H} H_{0}^{1}(\Omega),
\end{aligned}
$$

and hence $b(\cdot, \cdot)$ is a continuous nonsymmetric coercive bilinear functional on $\underset{\sim}{H}(\Omega) \times \underset{\sim}{H} H_{0}^{1}(\Omega)$. In addition, $\ell_{b}(\widehat{\varphi})(\cdot)$ is a continuous linear functional on $\underset{\sim}{V}$ for any $\varphi \in L_{M}^{2}(\Omega \times D)$.

For $r>d$, let

$$
{\underset{\sim}{Y}}^{r}:=\left\{\underset{\sim}{v} \in \underset{\sim}{L^{r}}(\Omega): \int_{\Omega} \underset{\sim}{v} \cdot \underset{\sim}{\nabla} \underset{\sim}{w} \mathrm{~d} \underset{\sim}{x}=0 \quad \forall \underset{\sim}{w} \in{\underset{\sim}{W}}^{1, \frac{r}{r-1}}(\Omega)\right\} .
$$

It is also convenient to rewrite $(3.24 \mathrm{~b})$ as

$$
a\left(\widehat{\psi}_{\varepsilon, L, \delta}^{n}, \widehat{\varphi}\right)=\ell_{a}\left({\underset{\sim}{u, L, \delta}}_{\varepsilon, \psi_{\varepsilon, L, \delta}^{n}}^{n}\right)(\widehat{\varphi}) \quad \forall \widehat{\varphi} \in \widehat{X},
$$

where, for all $\widehat{\varphi}_{1}, \widehat{\varphi}_{2} \in \widehat{X}$,

$$
\begin{aligned}
& a\left(\widehat{\varphi}_{1}, \widehat{\varphi}_{2}\right):=\int_{\Omega \times D} M\left(\widehat{\varphi}_{1} \widehat{\varphi}_{2}+\Delta t\left[\underset{\sim}{\varepsilon} \nabla_{x} \widehat{\varphi}_{1}-\underset{\sim}{u_{\varepsilon, L, \delta}^{n-1}} \widehat{\varphi}_{1}\right] \cdot \underset{\sim}{\nabla_{x}} \widehat{\varphi}_{2}\right. \\
& \left.+\frac{\Delta t}{2 \lambda} \underset{\sim}{\nabla_{q}} \widehat{\varphi}_{1} \cdot \underset{\sim}{\nabla_{q}} \widehat{\varphi}_{2}\right) \underset{\sim}{\mathrm{d} q} \mathrm{~d} x
\end{aligned}
$$

and, for all $\underset{\sim}{v} \in \underset{\sim}{H^{1}}(\Omega), \widehat{\eta} \in L_{M}^{2}(\Omega \times D)$ and $\widehat{\varphi} \in \widehat{X}$,

$$
\ell_{a}(\underset{\sim}{v}, \widehat{\eta})(\widehat{\varphi}):=\int_{\Omega \times D} M\left[\widehat{\psi}_{\varepsilon, L, \delta}^{n-1} \widehat{\varphi}+\Delta t \underset{\sim}{\sigma \underset{\sim}{\sigma} \underset{\sim}{q} \underset{\sim}{q}]} \beta_{\delta}^{L}(\widehat{\eta}) \cdot \underset{\sim}{\nabla_{q}} \widehat{\varphi}\right] \underset{\sim}{\mathrm{d} q} \underset{\sim}{\mathrm{d} x}
$$

It follows from (3.30) and (3.9) that for $r>d$

$$
\int_{\Omega \times D} M \underset{\sim}{v} \widehat{\varphi} \cdot \underset{\sim}{\nabla} \widehat{\varphi} \mathrm{d} \underset{\sim}{q} \mathrm{~d} \underset{\sim}{x}=0 \quad \forall \underset{\sim}{v} \in \underset{\sim}{Y}{ }^{r}, \quad \forall \widehat{\varphi} \in \widehat{X}
$$

and hence that $a(\cdot, \cdot)$ is a continuous nonsymmetric coercive bilinear functional on $\widehat{X} \times \widehat{X}$. In addition, $\ell_{a}(\underset{\sim}{v}, \widehat{\eta})(\cdot)$ is a linear functional on $\widehat{X}$ for all $\underset{\sim}{v} \in \underset{\sim}{H^{1}}(\Omega)$ and $\widehat{\eta} \in L_{M}^{2}(\Omega \times D)$.

In order to prove existence of a solution to $(3.24 \mathrm{a}, \mathrm{b})$, we consider a fixed-point argument. Given $\widehat{\psi} \in L_{M}^{2}(\Omega \times D)$ let $\left\{\sim_{\sim}^{\star}, \widehat{\psi}^{\star}\right\} \in \underset{\sim}{V} \times \widehat{X}$ be such that

$$
\begin{aligned}
& b\left(\underset{\sim}{u^{\star}}, \underset{\sim}{w}\right)=\ell_{b}(\widehat{\psi})(\underset{\sim}{w}) \quad \forall \underset{\sim}{w} \in \underset{\sim}{V}, \\
& a\left(\widehat{\psi}^{\star}, \widehat{\varphi}\right)=\ell_{a}\left(u_{\sim}^{\star}, \widehat{\psi}\right)(\widehat{\varphi}) \quad \forall \widehat{\varphi} \in \widehat{X} \text {. }
\end{aligned}
$$

The Lax-Milgram theorem yields the existence of a unique solution to $(3.34 \mathrm{a}, \mathrm{b})$, and so the overall procedure $(3.34 \mathrm{a}, \mathrm{b})$ is well defined.

Lemma 3.1. Let $G: L_{M}^{2}(\Omega \times D) \rightarrow \widehat{X} \subset L_{M}^{2}(\Omega \times D)$ denote the nonlinear map that takes $\widehat{\psi}$ to $\widehat{\psi}^{\star}=G(\widehat{\psi})$ via the procedure (3.34a,b). Then $G$ has a fixed point. Hence there exists a solution $\left\{\underset{\sim \varepsilon, L, \delta}{u_{n}^{n}}, \widehat{\psi}_{\varepsilon, L, \delta}^{n}\right\} \in \underset{\sim}{V} \times \widehat{X}$ to (3.24a,b). 
Proof. Clearly, a fixed point of $G$ yields a solution of (3.24a,b). In order to show that $G$ has a fixed point, we apply Schauder's fixed-point theorem; that is, we need to show that (i) $G: L_{M}^{2}(\Omega \times D) \rightarrow L_{M}^{2}(\Omega \times D)$ is continuous, that (ii) it is compact, and that (iii) there exists a $C_{\star} \in \mathbb{R}_{>0}$ such that

$$
\|\widehat{\psi}\|_{L_{M}^{2}(\Omega \times D)} \leq C_{\star}
$$

for every $\widehat{\psi} \in L_{M}^{2}(\Omega \times D)$ and $\kappa \in(0,1]$ satisfying $\widehat{\psi}=\kappa G(\widehat{\psi})$.

Let $\left\{\widehat{\psi}^{(i)}\right\}_{i \geq 0}$ be such that

$$
\widehat{\psi}^{(i)} \rightarrow \widehat{\psi} \quad \text { strongly in } L_{M}^{2}(\Omega \times D) \quad \text { as } i \rightarrow \infty .
$$

It follows immediately from (3.20) and (3.15) that

$$
\begin{aligned}
M^{\frac{1}{2}} \beta_{\delta}^{L}\left(\widehat{\psi}^{(i)}\right) & \rightarrow M^{\frac{1}{2}} \beta_{\delta}^{L}(\widehat{\psi}) & \text { strongly in } L^{\infty}(\Omega \times D) & \text { as } i \rightarrow \infty, \\
\underset{\approx}{C}\left(M \widehat{\psi}^{(i)}\right) & \rightarrow \underset{\approx}{C}(M \widehat{\psi}) & \text { strongly in } L^{2}(\Omega) & \text { as } i \rightarrow \infty .
\end{aligned}
$$

We need to show that

$$
\widehat{\eta}^{(i)}:=G\left(\widehat{\psi}^{(i)}\right) \rightarrow G(\widehat{\psi}) \quad \text { strongly in } L_{M}^{2}(\Omega \times D) \quad \text { as } i \rightarrow \infty,
$$

in order to prove (i) above. We have from the definition of $G$, see $(3.34 \mathrm{a}, \mathrm{b})$, that, for all $i \geq 0$,

$$
a\left(\widehat{\eta}^{(i)}, \widehat{\varphi}\right)=\ell_{a}\left({\underset{\sim}{v}}^{(i)}, \widehat{\psi}^{(i)}\right)(\widehat{\varphi}) \quad \forall \widehat{\varphi} \in \widehat{X},
$$

where ${\underset{\sim}{(i)}}^{(i)} \underset{\sim}{V}$ satisfies

$$
b\left(\underset{\sim}{v^{(i)}}, \underset{\sim}{w}\right)=\ell_{b}\left(\widehat{\psi}^{(i)}\right)(\underset{\sim}{w}) \quad \forall \underset{\sim}{\underset{\sim}{w}} \in \underset{\sim}{V} .
$$

Choosing $\widehat{\varphi}=\widehat{\eta}^{(i)}$ in (3.39a) yields, on noting the simple identity

$$
2\left(s_{1}-s_{2}\right) s_{1}=s_{1}^{2}+\left(s_{1}-s_{2}\right)^{2}-s_{2}^{2} \quad \forall s_{1}, s_{2} \in \mathbb{R},
$$

(3.33) and (3.20) that, for all $i \geq 0$,

$$
\begin{gathered}
\int_{\Omega \times D} M\left[\left|\widehat{\eta}^{(i)}\right|^{2}+\left|\widehat{\eta}^{(i)}-\widehat{\psi}_{\varepsilon, L, \delta}^{n-1}\right|^{2}+\frac{\Delta t}{2 \lambda}\left|\underset{\sim}{\nabla_{q}} \widehat{\eta}^{(i)}\right|^{2}+2 \varepsilon \Delta t\left|\underset{\sim}{\nabla_{x}} \eta^{(i)}\right|^{2}\right] \underset{\sim}{\mathrm{d} q \mathrm{~d} x} \\
\leq \int_{\Omega \times D} M\left|\widehat{\psi}_{\varepsilon, L, \delta}^{n-1}\right|^{2} \mathrm{~d} \underset{\sim}{\mathrm{d}} \underset{\sim}{\mathrm{d} x}+C(L, \lambda) \Delta t \int_{\Omega}\left|\underset{\sim}{\nabla_{x}}{\underset{\sim}{(i)}}^{(i)}\right|_{\sim}^{2} \underset{\sim}{\mathrm{d} x .}
\end{gathered}
$$

Choosing $\underset{\sim}{w} \equiv{\underset{\sim}{v}}^{(i)}$ in (3.39b), and noting (3.40), (3.29), (3.15), (3.2), a Poincaré inequality and (3.36) yields, for all $i \geq 0$, that

$$
\begin{aligned}
& \int_{\Omega}\left[\left.||_{\sim}^{(i)}\right|^{2}+\left|\underset{\sim}{v^{(i)}}-u_{\sim}^{n-1, L, \delta}\right|^{2}\right] \mathrm{d} x+\Delta t \nu \int_{\Omega}\left|\underset{\sim}{\nabla} \underset{\sim}{v_{\sim}^{(i)}}\right|^{2} \mathrm{~d} x \\
& \leq \int_{\Omega}\left|\underset{\sim}{u_{\varepsilon, L, \delta}^{n-1}}\right|^{2} \mathrm{~d} x+C \Delta t\left\|\underset{\sim}{S} \underset{\sim}{f_{\sim}^{n}}\right\|_{H^{1}(\Omega)}^{2}+C \Delta t \int_{\Omega \times D} M\left|\widehat{\psi}^{(i)}\right|^{2} \mathrm{~d} \underset{\sim}{\mathrm{d}} \underset{\sim}{\mathrm{d}} \leq C .
\end{aligned}
$$

Combining (3.41) and (3.42), we have for all $i \geq 0$ that

$$
\left\|\widehat{\eta}^{(i)}\right\|_{\widehat{X}}+\left\|{\underset{\sim}{(i)}}^{(i)}\right\|_{H^{1}(\Omega)} \leq C\left(L,(\Delta t)^{-1}\right) .
$$


It follows from (3.43), (3.9) and the compactness of the embedding (3.11b) that there exists a subsequence $\left\{\widehat{\eta}^{\left(i_{k}\right)},{\underset{\sim}{\left(i k_{k}\right)}}_{i_{k} \geq 0}\right.$ and functions $\widehat{\eta} \in \widehat{X}$ and $\underset{\sim}{v} \in \underset{\sim}{V}$ such that, as $i_{k} \rightarrow \infty$,

$$
\begin{array}{rlrl}
\widehat{\eta}^{\left(i_{k}\right)} & \rightarrow \widehat{\eta} & \text { weakly in } L^{s}\left(\Omega ; L_{M}^{2}(D)\right), \\
M^{\frac{1}{2}} \underset{\sim}{\nabla_{x} \widehat{\eta}^{\left(i_{k}\right)}} \rightarrow M^{\frac{1}{2}} \underset{\sim}{\nabla_{x} \widehat{\eta}} & \text { weakly in } \underset{\sim}{L^{2}(\Omega \times D),} \\
M^{\frac{1}{2}} \underset{\sim}{\nabla_{q} \widehat{\eta}^{\left(i_{k}\right)}} \rightarrow M^{\frac{1}{2}} \underset{\sim}{\nabla_{q} \widehat{\eta}} & \text { weakly in } \underset{\sim}{L^{2}(\Omega \times D),} \\
\widehat{\eta}^{\left(i_{k}\right)} \rightarrow \widehat{\eta} & \text { strongly in } \left.L_{M}^{2}(\Omega \times D)\right), \\
{\underset{\sim}{v}}^{\left(i_{k}\right)} \rightarrow \underset{\sim}{v} & \text { weakly in } \underset{\sim}{H^{1}}(\Omega) ;
\end{array}
$$

where $s \in[1, \infty)$ if $d=2$ or $s \in[1,6]$ if $d=3$. It follows from (3.39b), (3.28a,b), (3.44e) and (3.37b) that $\underset{\sim}{v} \in \underset{\sim}{V}$ and $\widehat{\psi} \in \widehat{X}$ satisfy

$$
b(\underset{\sim}{v}, \underset{\sim}{w})=\ell_{b}(\widehat{\psi})(\underset{\sim}{w}) \quad \forall \underset{\sim}{w} \in \underset{\sim}{V} .
$$

It follows from (3.39a), (3.32a,b), (3.44a-e) and (3.37a) that $\widehat{\eta}, \widehat{\psi} \in \widehat{X}$ and $\underset{\sim}{v} \in \underset{\sim}{V}$, satisfy

$$
a(\widehat{\eta}, \widehat{\varphi})=\ell_{a}(\underset{v}{v}, \widehat{\psi})(\widehat{\varphi}) \quad \forall \widehat{\varphi} \in \widehat{X} .
$$

Combining (3.46) and (3.45), we have that $\widehat{\eta}=G(\widehat{\psi}) \in \widehat{X}$. Therefore the whole sequence $\widehat{\eta}^{(i)} \equiv G\left(\widehat{\psi}^{(i)}\right) \rightarrow G(\widehat{\psi})$ strongly in $L_{M}^{2}(\Omega \times D)$ as $i \rightarrow \infty$, and so (i) holds.

As the embedding $\widehat{X} \hookrightarrow L_{M}^{2}(\Omega \times D)$ is compact, it follows that (ii) holds.

As regards (iii), $\widehat{\psi}=\kappa G(\widehat{\psi})$ implies that $\{\underset{\sim}{v}, \widehat{\psi}\} \in \underset{\sim}{V} \times \widehat{X}$ satisfies

$$
\begin{aligned}
& b(\underset{\sim}{v}, \underset{\sim}{w})=\ell_{b}(\widehat{\psi})(\underset{\sim}{w}) \quad \forall \underset{\sim}{w} \in \underset{\sim}{V}, \\
& a(\widehat{\psi}, \widehat{\varphi})=\kappa \ell_{a}(v, \widehat{\psi})(\widehat{\varphi}) \quad \forall \widehat{\varphi} \in \widehat{X} .
\end{aligned}
$$

Choosing $\underset{\sim}{w} \equiv \widehat{\sim}$ in (3.47a) yields, similarly to (3.42), that

$$
\begin{array}{r}
\frac{1}{2} \int_{\Omega}\left[|\underset{\sim}{v}|^{2}+\left|\underset{\sim}{v}-\underset{\sim}{u_{\varepsilon}^{n-1, \delta, \delta}}\right|^{2}-|\underset{\sim}{u, L, \delta}|^{2}\right] \mathrm{d} \underset{\sim}{x}+\Delta t \nu \int_{\Omega}\left|\underset{\sim}{\nabla_{x}} \underset{\sim}{v}\right|^{2} \mathrm{~d} x \\
=\Delta t\left[\left\langle{\underset{\sim}{f}}^{n}, \underset{\sim}{v}\right\rangle_{V}-k \mu \int_{\Omega} C(M \widehat{\psi}): \underset{\sim}{\nabla_{x}} \underset{\sim}{v} \mathrm{~d} x\right] .
\end{array}
$$

Choosing $\widehat{\varphi}=\left[\mathcal{F}_{\delta}^{L}\right]^{\prime}(\widehat{\psi})$ in (3.47b) and noting (3.18a), (3.20), (3.8), (1.4), (2.3a) and that $\underset{\sim}{v}$ is divergence-free yield

$$
\begin{aligned}
& \int_{\Omega \times D} M\left[\mathcal{F}_{\delta}^{L}(\widehat{\psi})-\mathcal{F}_{\delta}^{L}\left(\kappa \widehat{\psi}_{\varepsilon, L, \delta}^{n-1}\right)\right] \underset{\sim}{\mathrm{d} q \mathrm{~d} x} \\
& +\Delta t \int_{\Omega \times D} M\left[\varepsilon \underset{\sim}{\nabla} \widehat{\sim} \widehat{\psi} \cdot \underset{\sim}{\nabla_{x}}\left(\left[\mathcal{F}_{\delta}^{L}\right]^{\prime}(\widehat{\psi})\right)+\frac{1}{2 \lambda} \underset{\sim}{\nabla_{q}} \widehat{\psi} \cdot \underset{\sim}{\nabla_{q}}\left(\left[\mathcal{F}_{\delta}^{L}\right]^{\prime}(\widehat{\psi})\right)\right] \underset{\sim}{\mathrm{d} q \mathrm{~d} x} \\
& \leq \kappa \Delta t \int_{\Omega \times D} M \underset{\sim}{\sigma(v)} \underset{\sim}{q} \cdot \underset{\sim}{\nabla_{q}} \widehat{\psi} \mathrm{d} q \underset{\sim}{\sim} \mathrm{d} x \\
& =\kappa \Delta t \int_{\Omega} \underset{\approx}{\approx}(M \widehat{\psi}): \underset{\sim}{\sigma}(v) \mathrm{d} x \text {. }
\end{aligned}
$$


Combining (3.48) and (3.49), and noting (3.2) and a Poincaré inequality yields that

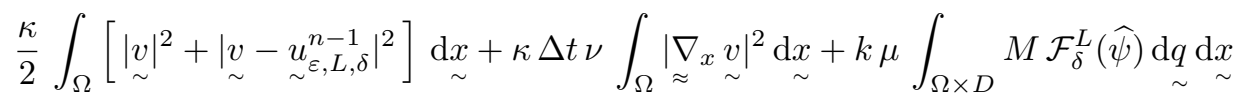

$$
\begin{aligned}
& +k \mu \Delta t \int_{\Omega \times D} M\left[\varepsilon \underset{\sim}{\varepsilon} \underset{x}{\nabla} \widehat{\psi} \cdot \underset{\sim}{\nabla_{x}}\left(\left[\mathcal{F}_{\delta}^{L}\right]^{\prime}(\widehat{\psi})\right)+\frac{1}{2 \lambda} \underset{\sim}{\nabla_{q}} \widehat{\psi} \cdot \underset{\sim}{\nabla_{q}}\left(\left[\mathcal{F}_{\delta}^{L}\right]^{\prime}(\widehat{\psi})\right)\right] \underset{\sim}{\mathrm{d} q} \mathrm{~d} \underset{\sim}{\sim}
\end{aligned}
$$

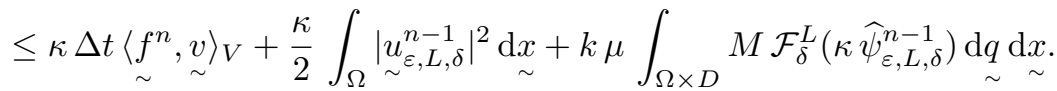

$$
\begin{aligned}
& \leq \frac{\kappa}{2} \Delta t \nu \int_{\Omega}|\underset{\approx}{\mid} \underset{\sim}{v}|^{2} \mathrm{~d} x+\kappa \Delta t C\left(\nu^{-1}\right) \underset{\sim}{S} \underset{\sim}{f^{n}} \|_{H^{1}(\Omega)}^{2} \\
& +\frac{\kappa}{2} \int_{\Omega}\left|u_{\sim}^{n-1}{ }_{\sim}^{n-1}\right|^{2} \mathrm{~d} x+k \mu \int_{\Omega \times D} M \mathcal{F}_{\delta}^{L}\left(\kappa \widehat{\psi}_{\varepsilon, L, \delta}^{n-1}\right) \underset{\sim}{\mathrm{d}} \underset{\sim}{\mathrm{d} x} .
\end{aligned}
$$

It is easy to show that $\mathcal{F}_{\delta}^{L}(s)$ is non-negative for all $s \in \mathbb{R}$, with $\mathcal{F}_{\delta}^{L}(1)=0$. Furthermore, for any $\kappa \in(0,1]$,

$$
\begin{array}{ll}
\mathcal{F}_{\delta}^{L}(\kappa s) \leq \mathcal{F}_{\delta}^{L}(s) & \text { if } s<0 \text { or } 1 \leq \kappa s \\
\mathcal{F}_{\delta}^{L}(\kappa s) \leq \mathcal{F}_{\delta}^{L}(0) \leq 1 & \text { if } 0 \leq \kappa s \leq 1
\end{array}
$$

Thus we deduce that

$$
\mathcal{F}_{\delta}^{L}(\kappa s) \leq \mathcal{F}_{\delta}^{L}(s)+1 \quad \forall s \in \mathbb{R}, \quad \forall \kappa \in(0,1]
$$

Hence, the bounds (3.50) and (3.51), on noting (3.19) and (3.18b), which implies that $\left[\mathcal{F}_{\delta}^{L}(s)\right]^{\prime \prime} \geq L^{-1}$ for all $s \in \mathbb{R}$, give rise to the desired bound (3.35) with $C_{*}$ dependent only on $L, k, \mu$ and $\widehat{\psi}_{\varepsilon, L, \delta}^{n-1}$. Hence (iii) holds, and so $G$ has a fixed point. Thus we have proved existence of a solution to $(3.24 \mathrm{a}, \mathrm{b})$.

Choosing $\underset{\sim}{w} \equiv u_{\varepsilon, L, \delta}^{n}$ in $(3.27)$ and $\widehat{\varphi} \equiv\left[\mathcal{F}_{\delta}^{L}\right]^{\prime}\left(\widehat{\psi}_{\varepsilon, L, \delta}^{n}\right)$, and combining, then yields, similarly to $(3.50)$, that

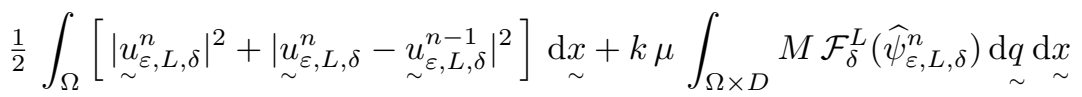

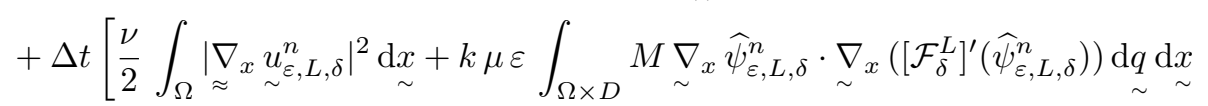

$$
\begin{aligned}
& \left.+\frac{1}{2 \lambda} \int_{\Omega \times D} M \underset{\sim}{\nabla_{q}} \widehat{\psi}_{\varepsilon, L, \delta}^{n} \cdot \underset{\sim}{\nabla_{q}}\left(\left[\mathcal{F}_{\delta}^{L}\right]^{\prime}\left(\widehat{\psi}_{\varepsilon, L, \delta}^{n}\right)\right) \underset{\sim}{\mathrm{d}} \underset{\sim}{\mathrm{d}} \underset{\sim}{x}\right] \\
& \leq \Delta t C\left(\nu^{-1}\right)\left\|\underset{\sim}{S} f_{\sim}^{n}\right\|_{H^{1}(\Omega)}^{2}+\frac{1}{2} \int_{\Omega}\left|\underset{\sim}{u_{\varepsilon, L, \delta}^{n-1}}\right|^{2} \mathrm{~d} x \\
& +k \mu \int_{\Omega \times D} M \mathcal{F}_{\delta}^{L}\left(\widehat{\psi}_{\varepsilon, L, \delta}^{n-1}\right) \underset{\sim}{q} \mathrm{~d} x \text {. }
\end{aligned}
$$


Summing (3.52) from $n=1 \rightarrow m$, with $1 \leq m \leq N$, yields that

$$
\begin{aligned}
& \frac{1}{2}\left[\int_{\Omega}\left|u_{\varepsilon, L, \delta}^{m}\right|^{2} \mathrm{~d} x+\sum_{\sim=1}^{m} \int_{\Omega}\left|u_{\varepsilon, L, \delta}^{n}-\underset{\sim}{u_{\varepsilon, L, \delta}^{n-1}}\right|^{2} \mathrm{~d} x\right]+k \mu \int_{\Omega \times D} M \mathcal{F}_{\delta}^{L}\left(\widehat{\psi}_{\varepsilon, L, \delta}^{m}\right) \mathrm{d} \underset{\sim}{\sim} \mathrm{d} x \\
& +\sum_{n=1}^{m} \Delta t\left[\frac{\nu}{2} \int_{\Omega}\left|\underset{\sim}{\nabla_{x}} \underset{\sim}{u_{\varepsilon, L, \delta}^{n}}\right|^{2} \mathrm{~d} x+k \mu \varepsilon \int_{\Omega \times D} M \underset{\sim}{\underset{\sim}{\nabla}} \widehat{\psi}_{\varepsilon, L, \delta}^{n} \cdot \underset{\sim}{\nabla_{x}}\left(\left[\mathcal{F}_{\delta}^{L}\right]^{\prime}\left(\widehat{\psi}_{\varepsilon, L, \delta}^{n}\right)\right) \mathrm{d} q \underset{\sim}{\mathrm{d} x}\right. \\
& \left.+\frac{1}{2 \lambda} \int_{\Omega \times D} \underset{\sim}{\underset{\sim}{\nabla}} \widehat{\psi}_{\varepsilon, L, \delta}^{n} \cdot \underset{\sim}{\nabla_{q}}\left(\left[\mathcal{F}_{\delta}^{L}\right]^{\prime}\left(\widehat{\psi}_{\varepsilon, L, \delta}^{n}\right)\right) \mathrm{d} \underset{\sim}{\mathrm{d} x} \underset{\sim}{\sim}\right] \\
& \leq \frac{1}{2} \int_{\Omega}\left|u_{\sim}^{0}\right|^{2} \mathrm{~d} x+k \mu \int_{\Omega \times D} M \mathcal{F}_{\delta}^{L}\left(\widehat{\psi}_{0}\right) \underset{\sim}{\mathrm{d} q} \mathrm{~d} x+C\left(\nu^{-1}\right) \sum_{n=1}^{m} \Delta t\|\|_{\sim} \underset{\sim}{f_{\sim}^{n}} \|_{H^{1}(\Omega)}^{2} \\
& \leq \frac{1}{2} \int_{\Omega}\left|u_{\sim}^{0}\right|^{2} \mathrm{~d} x+k \mu \int_{\Omega \times D} M \mathcal{F}_{\delta}^{L}\left(\widehat{\psi}_{0}\right) \underset{\sim}{\mathrm{d} q} \underset{\sim}{\mathrm{d} x}+C\left(\nu^{-1}\right) \int_{0}^{t_{m}}\|\underset{\sim}{S} \underset{\sim}{f}\|_{H^{1}(\Omega)}^{2} \mathrm{~d} t \leq C ;
\end{aligned}
$$

where $C$ is independent of $\delta, L$ and $\Delta t$, on assuming that $L$ is chosen so that

$$
0 \leq \widehat{\psi}_{0} \leq L \quad \text { a.e. in } \Omega \times D .
$$

Choosing $\widehat{\varphi}=\widehat{\psi}_{\varepsilon, L, \delta}^{n}$ in (3.31), and noting (3.40), (3.33), (3.20) and (1.5), yields that

$$
\begin{aligned}
& \int_{\Omega \times D} M\left[\left|\widehat{\psi}_{\varepsilon, L, \delta}^{n}\right|^{2}+\left|\widehat{\psi}_{\varepsilon, L, \delta}^{n}-\widehat{\psi}_{\varepsilon, L, \delta}^{n-1}\right|^{2}\right] \underset{\sim}{\mathrm{d}} \underset{\sim}{q} \mathrm{~d} x \\
& +\Delta t \int_{\Omega \times D} M\left[2 \varepsilon\left|{\underset{\sim}{x}}_{x} \widehat{\psi}_{\varepsilon, L, \delta}^{n}\right|^{2}+\frac{1}{\lambda}\left|{\underset{\sim}{q}}_{q} \widehat{\psi}_{\varepsilon, L, \delta}^{n}\right|^{2}\right] \underset{\sim}{\mathrm{d}} \underset{\sim}{\mathrm{d} x} \\
& \left.\left.=\int_{\Omega \times D} M\left[\left|\widehat{\psi}_{\varepsilon, L, \delta}^{n-1}\right|^{2}+\Delta t \underset{\sim}{\sigma\left(u_{\sim}^{n}, L, \delta\right.}\right) \underset{\sim}{q}\right] \beta_{\delta}^{L}\left(\widehat{\psi}_{\varepsilon, L, \delta}^{n}\right) \cdot \underset{\sim}{\nabla_{q}} \widehat{\psi}_{\varepsilon, L, \delta}^{n}\right] \underset{\sim}{\mathrm{d} q} \mathrm{~d} x \\
& \leq \int_{\Omega \times D} M\left|\widehat{\psi}_{\varepsilon, L, \delta}^{n-1}\right|^{2} \mathrm{~d} q \underset{\sim}{\mathrm{d} x}+\frac{\Delta t}{2 \lambda} \int_{\Omega \times D} M\left|\underset{\sim}{\nabla_{q}} \widehat{\psi}_{\varepsilon, L, \delta}^{n}\right|^{2} \underset{\sim}{\mathrm{d} q} \mathrm{~d} x \\
& +C(L, \lambda) \Delta t \int_{\Omega}\left|\nabla_{\approx} \underset{\sim}{u_{\varepsilon, L, \delta}^{n}}\right|^{2} \mathrm{~d} x .
\end{aligned}
$$

Summing (3.55) from $n=1 \rightarrow m$, with $1 \leq m \leq N$, yields, on noting (3.53), that

$$
\begin{aligned}
& \int_{\Omega \times D} M\left|\widehat{\psi}_{\varepsilon, L, \delta}^{m}\right|^{2} \mathrm{~d} \underset{\sim}{q} \mathrm{~d} x+\sum_{n=1}^{m} \int_{\Omega \times D} M\left|\widehat{\psi}_{\varepsilon, L, \delta}^{n}-\widehat{\psi}_{\varepsilon, L, \delta}^{n-1}\right|^{2} \mathrm{~d} \underset{\sim}{q} \mathrm{~d} x \\
& +\sum_{n=1}^{m} \Delta t \int_{\Omega \times D} M\left[2 \varepsilon\left|\underset{\sim}{\nabla_{x}} \widehat{\psi}_{\varepsilon, L, \delta}^{n}\right|^{2}+\frac{1}{2 \lambda}\left|\underset{\sim}{\nabla_{q}} \widehat{\psi}_{\varepsilon, L, \delta}^{n}\right|^{2}\right] \underset{\sim}{\mathrm{d}} \underset{\sim}{\mathrm{d} x} \\
& \leq \int_{\Omega \times D} M\left|\widehat{\psi}_{0}\right|^{2} \mathrm{~d} q \underset{\sim}{\mathrm{d}} \underset{\sim}{x}+C(L) \sum_{n=1}^{m} \Delta t \int_{\Omega}\left|\underset{\sim}{\nabla_{x}} \underset{\sim}{u_{\varepsilon, L, \delta}^{n}}\right|^{2} \mathrm{~d} x \leq C(L) .
\end{aligned}
$$

Choosing $\underset{\sim}{w} \equiv \underset{\sim}{S}\left(\frac{u_{\varepsilon, L, \delta}^{n}-u_{\varepsilon, L, \delta}^{n-1}}{\Delta t}\right) \in \underset{\sim}{V}$ in (3.27) yields, on noting (3.2), (3.3) and 
(3.29), that

$$
\begin{aligned}
& \int_{\Omega}\left[\left|\nabla_{x}\left[\underset{\sim}{S}\left(\frac{u_{\varepsilon, L, \delta}^{n}-u_{\mathcal{\sim}, L, \delta}^{n-1}}{\Delta t}\right)\right]\right|^{2}+\left|\underset{\sim}{S}\left(\frac{\underset{\sim}{u_{\varepsilon, L, \delta}^{n}-u_{\sim \varepsilon, L, \delta}^{n-1}}}{\Delta t}\right)\right|^{2}\right] \underset{\sim}{\sim}
\end{aligned}
$$

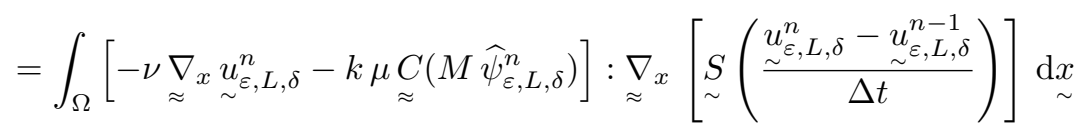

$$
\begin{aligned}
& +\int_{\Omega^{\sim}} u_{\varepsilon, L, \delta}^{n} \cdot\left[\left(\underset{\sim}{u_{\varepsilon, L, \delta}^{n-1}} \cdot \underset{\sim}{\nabla_{x}}\right)\left[\underset{\sim}{S}\left(\frac{u_{\varepsilon, L, \delta}^{n}-u_{\sim, L, \delta}^{n-1}}{\Delta t}\right)\right]\right] \mathrm{d} x+\left\langle f_{\sim}^{n}, \underset{\sim}{S}\left(\frac{u_{\varepsilon, L, \delta}^{n}-u_{\mathcal{\sim}, L, \delta}^{n-1}}{\Delta t}\right)\right\rangle_{V}
\end{aligned}
$$

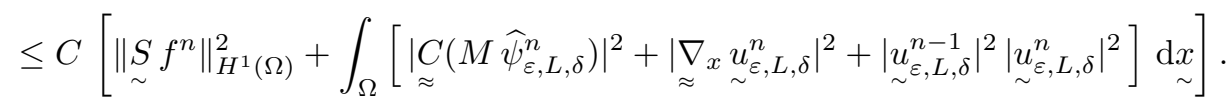

Applying the Cauchy-Schwarz inequality, the algebraic-geometric mean inequality, (3.4), and a Poincaré inequality yields that

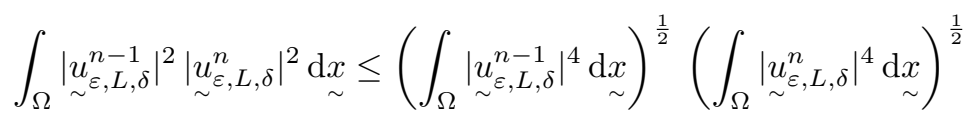

$$
\begin{aligned}
& \leq \frac{1}{2} \sum_{m=n-1}^{n} \int_{\Omega}\left|\underset{\sim}{u_{\varepsilon, L, \delta}^{m}}\right|^{4} \mathrm{~d} x
\end{aligned}
$$

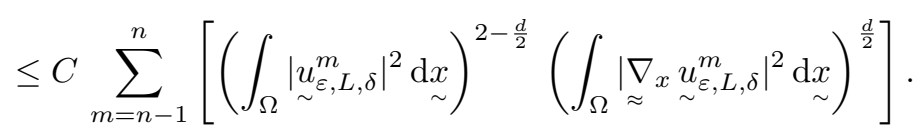

Taking the $\frac{2}{d}$ power of both sides of (3.57), summing from $n=1 \rightarrow N$, and noting (3.58), (3.15), (3.56), (3.53) and (3.23) yields that

$$
\begin{aligned}
& \sum_{n=1}^{N} \Delta t\left(\int_{\Omega}\left[\left|\nabla_{x}\left[\underset{\sim}{S}\left(\frac{u_{\varepsilon, L, \delta}^{n}-u_{\varepsilon, L, \delta}^{n-1}}{\Delta t}\right)\right]\right|^{2}+\left|\underset{\sim}{S}\left(\frac{\underset{\sim}{u_{\varepsilon, L, \delta}^{n}-u_{\varepsilon, L, \delta}^{n-1}}}{\Delta t}\right)\right|^{2}\right] \underset{\sim}{\mathrm{d} x}\right)^{\frac{2}{d}} \\
& \leq C\left[\sum_{n=1}^{N} \Delta t\left(\int_{\Omega}\left|C\left(M \widehat{\psi}_{\varepsilon, L, \delta}^{n}\right)\right|^{2} \mathrm{~d} \underset{\sim}{x}\right)^{\frac{2}{d}}\right]+C(T)\left[\sum_{n=1}^{N} \Delta t \int_{\Omega}\left|\underset{\approx}{\nabla_{x}} \underset{\sim}{u_{\varepsilon, L, \delta}^{n}}\right|^{2} \mathrm{~d} x\right]_{\sim}^{\frac{2}{d}} \\
& +C(T)\left[\max _{n=0 \rightarrow N}\left(\int_{\Omega} \mid \underset{\sim}{\left.u_{\varepsilon, L, \delta}^{n}\right|^{2} \mathrm{~d} x} \sim\right)^{\frac{4}{d}-1}\right]\left[\sum_{n=0}^{N} \Delta t \int_{\Omega}|\underset{\approx}{\mid} \underbrace{}_{x} \underset{\sim}{u_{\varepsilon, L, \delta}^{n}}|^{2} \mathrm{~d} x\right] \\
& +\sum_{n=1}^{N} \Delta t\left\|\underset{\sim}{S f_{\sim}^{n}}\right\|_{H^{1}(\Omega)}^{\frac{4}{d}} \\
& \leq C(L, T)+C \int_{0}^{T}\|\underset{\sim}{S f}\|_{H^{1}(\Omega)}^{\frac{4}{d}} \mathrm{~d} t \leq C(L, T) .
\end{aligned}
$$


Choosing $\widehat{\varphi} \equiv \mathcal{G}\left(\frac{\widehat{\psi}_{\varepsilon, L, \delta}^{n}-\widehat{\psi}_{\varepsilon, L, \delta}^{n-1}}{\Delta t}\right) \in \widehat{X}$ in (3.31) yields, on noting (3.12), (3.13), (3.20) and (1.5), that

$$
\begin{aligned}
& \left\|\mathcal{G}\left(\frac{\widehat{\psi}_{\varepsilon, L, \delta}^{n}-\widehat{\psi}_{\varepsilon, L, \delta}^{n-1}}{\Delta t}\right)\right\|_{\widehat{X}}^{2} \\
& \leq C\left[\left\|\widehat{\psi}_{\varepsilon, L, \delta}^{n}\right\|_{\widehat{X}}^{2}+\left\|u_{\sim}^{n} u_{\varepsilon, L}^{n}\right\|_{H^{1}(\Omega)}^{2}+\left.\int_{\Omega \times D} M\left|\underset{\sim}{\left.u_{\varepsilon, L, \delta}^{n-1}\right|^{2}}\right| \widehat{\psi}_{\varepsilon, L, \delta}^{n}\right|^{2} \mathrm{~d} \underset{\sim}{\mathrm{d} x} \underset{\sim}{\mathrm{d}}\right] .
\end{aligned}
$$

Similarly to (3.58), on noting (3.4) and (3.10), we have that

$$
\begin{aligned}
& \left.\int_{\Omega \times D} M\left|\underset{\sim}{\left.u_{\varepsilon, L, \delta}^{n-1}\right|^{2}}\right| \widehat{\psi}_{\varepsilon, L, \delta}^{n}\right|^{2} \mathrm{~d} \underset{\sim}{q \mathrm{~d} x} \leq\left\|\underset{\sim}{u_{\varepsilon, L, \delta}^{n-1}}\right\|_{L^{4}(\Omega)}^{2}\left\|\widehat{\psi}_{\varepsilon, L, \delta}^{n}\right\|_{L^{4}\left(\Omega ; L_{M}^{2}(D)\right)}^{2} \\
& \leq C\left[\| u_{\sim}^{n-1, \delta}\left[\left\|_{L^{2}(\Omega)}^{4-d}\right\|{\underset{\sim}{x}}_{x} \underset{\sim}{u_{\varepsilon, L, \delta}^{n-1} \|_{L^{2}(\Omega)}^{d}}\right.\right. \\
& \left.+\left\|\widehat{\psi}_{\varepsilon, L, \delta}^{n}\right\|_{L^{2}\left(\Omega ; L_{M}^{2}(D)\right)}^{4-d}\left\|\widehat{\psi}_{\varepsilon, L, \delta}^{n}\right\|_{H^{1}\left(\Omega ; L_{M}^{2}(D)\right)}^{d}\right] .
\end{aligned}
$$

Taking the $\frac{2}{d}$ power of both sides of (3.60), summing from $n=1 \rightarrow N$, and noting (3.61), (3.56) and (3.23) yields, similarly to (3.59), that

$$
\sum_{n=1}^{N} \Delta t\left\|\mathcal{G}\left(\frac{\widehat{\psi}_{\varepsilon, L, \delta}^{n}-\widehat{\psi}_{\varepsilon, L, \delta}^{n-1}}{\Delta t}\right)\right\|_{\widehat{X}}^{\frac{4}{d}} \leq C(L, T) .
$$

Now we introduce some definitions prior to passing to the limit $\Delta t \rightarrow 0_{+}$. Let

$$
u_{\sim, L, \delta}^{\Delta t}(\cdot, t):=\frac{t-t_{n-1}}{\Delta t} u_{\sim, L, \delta}^{n}(\cdot)+\frac{t_{n}-t}{\Delta t} u_{\sim, L, \delta}^{n-1}(\cdot), \quad t \in\left[t_{n-1}, t_{n}\right], \quad n \geq 1,
$$

and

$$
u_{\sim, L, \delta}^{\Delta t,+}(\cdot, t):=\sim_{\sim}^{n}(\cdot), \quad u_{\varepsilon, L, \delta}^{\Delta t,-}(\cdot, t):={\underset{\sim}{u}}^{n-1}(\cdot), \quad t \in\left(t_{n-1}, t_{n}\right], \quad n \geq 1 .
$$

We note for future reference that

$$
u_{\varepsilon, L, \delta}^{\Delta t}-{\underset{\sim}{\varepsilon, L, \delta}}_{\varepsilon t, \pm}^{\Delta t,}=\left(t-t_{n}^{ \pm}\right) \frac{\partial u_{\varepsilon, L, \delta}^{\Delta t}}{\partial t}, \quad t \in\left(t_{n-1}, t_{n}\right), \quad n \geq 1
$$

where $t_{n}^{+}:=t_{n}$ and $t_{n}^{-}:=t_{n-1}$. Using the above notation, and introducing analogous notation for $\left\{\widehat{\psi}_{\varepsilon, L, \delta}^{n}, f_{\sim}^{n}\right\}_{n=0}^{N},(3.27)$ summed for $n=1 \rightarrow N$ can be restated as

$$
\begin{aligned}
& \int_{0}^{T}\left\langle\frac{\partial u_{\sim}^{\Delta t} t, \delta}{\partial t}, \underset{\sim}{w}\right\rangle_{V} \mathrm{~d} t \\
& +\int_{0}^{T} \int_{\Omega}\left[\left[\left(\underset{\sim}{u_{\varepsilon, L, \delta}^{\Delta t,-}} \cdot \underset{\sim}{\nabla} x\right) \underset{\sim}{\sim_{\varepsilon, L, \delta}}\right] \cdot \underset{\sim}{w}+\underset{\sim}{\underset{\sim}{\nabla}} \underset{\sim}{u_{\sim}} \underset{\varepsilon, L, \delta}{\Delta t,+}: \underset{\sim}{\nabla_{x}} \underset{\sim}{w}\right] \mathrm{d} \underset{\sim}{\mathrm{d}} \mathrm{d} t \\
& \left.=\int_{0}^{T}\left[\langle\underset{\sim}{f} \underset{\sim}{\Delta t,+}, \underset{\sim}{w}\rangle_{V}-k \mu \int_{\Omega} \underset{\approx}{C}\left(M \widehat{\psi}_{\varepsilon, L, \delta}^{\Delta t,+}\right): \underset{\approx}{\nabla_{x}} \underset{\sim}{w} \mathrm{~d} x\right] \mathrm{\sim}\right] \quad \forall \underset{\sim}{\forall} \in L^{\frac{4}{4-d}}(0, T ; \underset{\sim}{V}) .
\end{aligned}
$$


Similarly, (3.31) summed for $n=1 \rightarrow N$ can be restated as

$$
\begin{aligned}
& \int_{0}^{T}\left\langle M \frac{\partial \widehat{\psi} \widehat{\psi}_{\varepsilon, L, \delta}^{\Delta t}}{\partial t}, \widehat{\varphi}\right\rangle_{\widehat{X}} \underset{\sim}{\mathrm{d}} \underset{\sim}{\mathrm{d}} \underset{\sim}{x} \mathrm{~d} t
\end{aligned}
$$

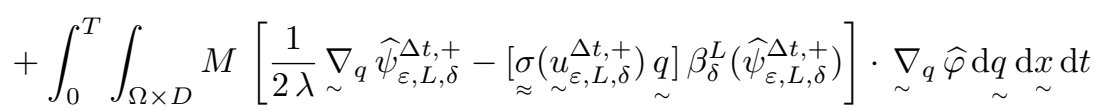

$$
\begin{aligned}
& +\int_{0}^{T} \int_{\Omega \times D} M\left[\underset{\sim}{\varepsilon} \underset{x}{\nabla_{x}} \widehat{\psi}_{\varepsilon, L, \delta}^{\Delta t,+}-\underset{\sim}{u_{\varepsilon, L, \delta}^{\Delta t,-}} \widehat{\psi}_{\varepsilon, L, \delta}^{\Delta t,+}\right] \cdot \underset{\sim}{\nabla_{x}} \widehat{\varphi} \underset{\sim}{\mathrm{d}} \underset{\sim}{\mathrm{d}} \underset{\sim}{x} \mathrm{~d} t=0 \\
& \forall \widehat{\varphi} \in L^{2}(0, T ; \widehat{X}) .
\end{aligned}
$$

We have from (3.53) and (3.63a,b), on noting (3.18b), that

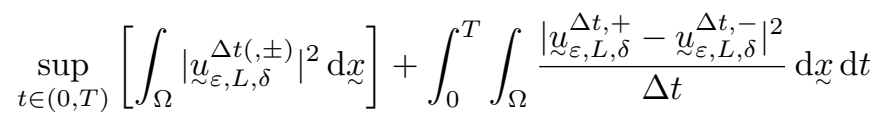

$$
\begin{aligned}
& +\nu \int_{0}^{T} \int_{\Omega}\left|\underset{\approx}{\nabla_{x}} \underset{\sim \varepsilon, L, \delta}{\Delta t(, \pm)}\right|^{2} \mathrm{~d} \underset{\sim}{x} \mathrm{~d} t \leq C(T)
\end{aligned}
$$

In the above, the notation ${\underset{\sim}{\varepsilon, L, \delta}}_{\varepsilon t(, \pm)}$ means $\underset{\underset{\varepsilon}{u}, L, \delta}{\Delta t}$ with or without the superscripts \pm . Similarly, we have from (3.56), (3.53), (3.19), (3.15), (3.59), (3.62) and (3.63a,b) that

$$
\begin{aligned}
& \sup _{t \in(0, T)}\left[\int_{\Omega \times D} M\left|\widehat{\psi}_{\varepsilon, L, \delta}^{\Delta t(, \pm)}\right|^{2} \mathrm{~d} \underset{\sim}{\operatorname{d} x}\right]+\frac{1}{\delta} \sup _{t \in(0, T)}\left[\int_{\Omega \times D} M\left[\widehat{\psi}_{\varepsilon, L, \delta}^{\Delta t(, \pm)}\right]_{-}^{2} \underset{\sim}{\mathrm{d} q} \mathrm{~d} x\right] \\
& +\frac{1}{\lambda} \int_{0}^{T} \int_{\Omega \times D} M\left|{\underset{\sim}{q}}_{q} \widehat{\psi}_{\varepsilon, L, \delta}^{\Delta t,+}\right|^{2} \underset{\sim}{\mathrm{d} q} \underset{\sim}{\mathrm{d}} \underset{\sim}{x} \mathrm{~d} t+\varepsilon \int_{0}^{T} \int_{\Omega \times D} M\left|\underset{\sim}{\nabla_{x}} \widehat{\psi}_{\varepsilon, L, \delta}^{\Delta t,+}\right|^{2} \mathrm{~d} \underset{\sim}{\mathrm{d}} \mathrm{d} \underset{\sim}{x} \mathrm{~d} t \\
& +\sup _{t \in(0, T)}\left[\int_{\Omega}\left|C\left(M \widehat{\psi}_{\varepsilon, L, \delta}^{\Delta t(, \pm)}\right)\right|^{2} \mathrm{~d} x\right]+\int_{0}^{T} \int_{\Omega \times D} M \frac{\left|\widehat{\psi}_{\varepsilon, L, \delta}^{\Delta t,+}-\widehat{\psi}_{\varepsilon, L, \delta}^{\Delta t,-}\right|^{2}}{\Delta t} \mathrm{~d} \underset{\sim}{q} \mathrm{~d} \underset{\sim}{x} \mathrm{~d} t \\
& \left.+\int_{0}^{T} \int_{\Omega \times D} M\left[\left\|\underset{\sim}{S \frac{\partial u_{\varepsilon, L, \delta}^{\Delta t}}{\partial t}}\right\|_{H^{1}(\Omega)}^{\frac{4}{d}}+\left\|\mathcal{G} \frac{\partial \widehat{\psi}_{\varepsilon, L, \delta}^{\Delta t}}{\partial t}\right\|_{\widehat{X}}\right] \underset{\sim}{\frac{4}{d}}\right] \underset{\sim}{\operatorname{d}} \underset{\sim}{\mathrm{d} t} \leq C(L, T) .
\end{aligned}
$$

We are now in a position to prove the following convergence result.

Lemma 3.2. There exists a subsequence of $\left\{{\underset{\sim}{u, L, \delta}, \Delta t}_{\mathcal{L}, t}^{\Delta t} \widehat{\psi}_{\varepsilon, L, \delta}^{\Delta t}\right\}_{\Delta t>0}$, and functions ${\underset{\sim}{\varepsilon, L, \delta}}_{\varepsilon} \in L^{\infty}\left(0, T ; \underset{\sim}{L^{2}}(\Omega)\right) \cap L^{2}(0, T ; \underset{\sim}{V}) \cap W^{1, \frac{4}{d}}\left(0, T ; V^{\prime}\right)$ and $\widehat{\psi}_{\varepsilon, L, \delta} \in$ 


$$
\begin{aligned}
& L^{\infty}\left(0, T ; L_{M}^{2}(\Omega \times D)\right) \cap L^{2}(0, T ; \widehat{X}) \cap H^{1}\left(0, T ; \widehat{X}^{\prime}\right) \text { such that, as } \Delta t \rightarrow 0_{+} \text {, } \\
& u_{\sim, L, \delta}^{\Delta t(, \pm)} \rightarrow u_{\sim, L, \delta} \quad \text { weak }{ }^{*} \text { in } L^{\infty}\left(0, T ; L_{\sim}^{2}(\Omega)\right), \\
& {\underset{\sim}{\varepsilon, L, \delta}}_{\varepsilon t(, \pm)}^{\Delta t} \rightarrow u_{\varepsilon, L, \delta} \quad \text { weakly in } L^{2}(0, T ; V) \text {, } \\
& S \frac{\partial u_{\varepsilon, L, \delta}^{\Delta t}}{\partial t} \rightarrow S \frac{\partial u_{\varepsilon, L, \delta}}{\partial t} \quad \text { weakly in } L^{\frac{4}{d}}(0, T ; V) \text {, } \\
& u_{\sim, L, \delta}^{\Delta t(, \pm)} \rightarrow u_{\varepsilon, L, \delta} \quad \text { strongly in } L^{2}\left(0, T ; L_{\sim}^{r}(\Omega)\right),
\end{aligned}
$$

where $r \in[1, \infty)$ if $d=2$ and $r \in[1,6)$ if $d=3$; and

$$
\begin{aligned}
& M^{\frac{1}{2}} \widehat{\psi}_{\varepsilon, L, \delta}^{\Delta t(, \pm)} \rightarrow M^{\frac{1}{2}} \widehat{\psi}_{\varepsilon, L, \delta} \quad \text { weak }^{*} \text { in } L^{\infty}\left(0, T ; L^{2}(\Omega \times D)\right), \\
& M^{\frac{1}{2}} \underset{\sim}{\nabla_{q}} \widehat{\psi}_{\varepsilon, L, \delta}^{\Delta t,+} \rightarrow M^{\frac{1}{2}} \underset{\sim}{\nabla_{q}} \widehat{\psi}_{\varepsilon, L, \delta} \quad \text { weakly in } L^{2}\left(0, T ; \underset{\sim}{L^{2}}(\Omega \times D)\right), \\
& M^{\frac{1}{2}} \underset{\sim}{\nabla_{x}} \widehat{\psi}_{\varepsilon, L, \delta}^{\Delta t,+} \rightarrow M^{\frac{1}{2}} \underset{\sim}{\nabla_{x}} \widehat{\psi}_{\varepsilon, L, \delta} \quad \text { weakly in } L^{2}\left(0, T ; \underset{\sim}{L^{2}}(\Omega \times D)\right), \\
& \mathcal{G} \frac{\partial \widehat{\psi}_{\varepsilon, L, \delta}^{\Delta t}}{\partial t} \rightarrow \mathcal{G} \frac{\partial \widehat{\psi}_{\varepsilon, L, \delta}}{\partial t} \quad \text { weakly in } L^{\frac{4}{d}}(0, T ; \widehat{X}) \\
& M^{\frac{1}{2}} \widehat{\psi}_{\varepsilon, L, \delta}^{\Delta t(, \pm)} \rightarrow M^{\frac{1}{2}} \widehat{\psi}_{\varepsilon, L, \delta} \quad \text { strongly in } L^{2}\left(0, T ; L^{2}(\Omega \times D)\right), \\
& M^{\frac{1}{2}} \beta_{\delta}^{L}\left(\widehat{\psi}_{\varepsilon, L, \delta}^{\Delta t(, \pm)}\right) \rightarrow M^{\frac{1}{2}} \beta_{\delta}^{L}\left(\widehat{\psi}_{\varepsilon, L, \delta}\right) \quad \text { strongly in } L^{\infty}\left(0, T ; L^{\infty}(\Omega \times D)\right) \\
& \underset{\approx}{C}\left(M \widehat{\psi}_{\varepsilon, L, \delta}^{\Delta t(, \pm)}\right) \rightarrow \underset{\approx}{C}\left(M \widehat{\psi}_{\varepsilon, L, \delta}\right) \quad \text { strongly in } L^{2}\left(0, T ; \underset{\approx}{L^{2}}(\Omega)\right) .
\end{aligned}
$$

Proof. The results $(3.69 \mathrm{a}-\mathrm{c})$ follow immediately from the bounds (3.67) and the bound on $u_{\varepsilon, L, \delta}^{\Delta t}$ in (3.68). The strong convergence result (3.69d) for $u_{\varepsilon, L, \delta}^{\Delta t}$ follows immediately from (3.69a-c), (3.3) and (3.14), on noting that $\underset{\sim}{V} \subset \underset{\sim}{H}(\Omega)$ is compactly embedded in ${\underset{\sim}{r}}^{r}(\Omega)$ for the stated values of $r$. We now prove (3.69d) for ${\underset{\sim}{u}, L, \delta}_{\varepsilon t, \pm}^{\Delta t}$. First, we obtain from the bound on the second term on the left-hand side of (3.67) and from (3.64) that

$$
\left\|u_{\varepsilon, L, \delta}^{\Delta t}-{\underset{\sim}{u, L, \delta}}_{\varepsilon, \delta}^{\Delta t, \pm}\right\|_{L^{2}\left(0, T, L^{2}(\Omega)\right)}^{2} \leq C \Delta t .
$$

Second, we note from (3.4) that, for all $\eta \in L^{2}\left(0, T ; H^{1}(\Omega)\right)$,

$$
\|\eta\|_{L^{2}\left(0, T ; L^{r}(\Omega)\right)} \leq C\|\eta\|_{L^{2}\left(0, T ; L^{2}(\Omega)\right)}^{1-\theta}\|\eta\|_{L^{2}\left(0, T ; H^{1}(\Omega)\right)}^{\theta}
$$

for any $r \in[2, \infty)$ if $d=2$ or any $r \in[2,6)$ if $d=3$, where $\theta=d\left(\frac{1}{2}-\frac{1}{r}\right) \in[0,1)$. Hence, combining (3.71), (3.72), and (3.69d) for ${\underset{\sim}{\varepsilon, L, \delta}}_{\varepsilon t}^{\Delta t}$ yields (3.69d) for ${\underset{\sim}{\varepsilon, L, \delta}}_{\varepsilon,}^{\Delta t, \pm}$.

The result (3.70a) follows immediately from the bounds on the first and sixth terms on the left-hand side of (3.68). It follows immediately from the bound on the third term on the left-hand side of (3.68) that (3.70b) holds for some limit $g \in L^{2}\left(0, T ; L^{2}(\Omega \times D)\right)$, which we need to identify. However, for any $\underset{\sim}{\eta} \in L^{2}\left(0, T ; C_{0}^{\infty}(\Omega \times D)\right)$, it follows from (1.4) and the compact support of $\underset{\sim}{\eta}$ on $D$ 
that $\left[\nabla_{q} \cdot\left(M^{\frac{1}{2}} \eta\right)\right] / M^{\frac{1}{2}} \in L^{2}\left(0, T ; L^{2}(\Omega \times D)\right)$, and hence the above convergence implies, noting (3.70a), that

$$
\begin{aligned}
\int_{0}^{T} \int_{\Omega \times D} \underset{\sim}{g} \cdot \underset{\sim}{\eta} \mathrm{d} q \underset{\sim}{\mathrm{d} x} \underset{\sim}{\mathrm{d} t} & \leftarrow-\int_{0}^{T} \int_{\Omega \times D} M^{\frac{1}{2}} \widehat{\psi}_{\varepsilon, L, \delta}^{\Delta t,+} \frac{\nabla_{q} \cdot\left(M^{\frac{1}{2}} \underset{\sim}{\eta}\right)}{M^{\frac{1}{2}}} \mathrm{~d} \underset{\sim}{\mathrm{d}} \mathrm{d} \underset{\sim}{x} \mathrm{~d} t \\
& \rightarrow-\int_{0}^{T} \int_{\Omega \times D} M^{\frac{1}{2}} \widehat{\psi}_{\varepsilon, L, \delta} \frac{\nabla_{q} \cdot\left(M^{\frac{1}{2}} \underset{\sim}{\eta}\right)}{M^{\frac{1}{2}}} \mathrm{~d} \underset{\sim}{\mathrm{d}} \underset{\sim}{x} \mathrm{~d} t
\end{aligned}
$$

as $\Delta t \rightarrow 0_{+}$. Hence the desired result (3.70b) follows from (3.73), noting the denseness of $C_{0}^{\infty}(\Omega \times D)$ in $L^{2}(\Omega \times D)$. Similar arguments prove $(3.70 \mathrm{c}, \mathrm{d})$ on noting (3.70a), and the fourth and seventh bounds in (3.68). The strong convergence result (3.70e) for $\widehat{\psi}_{\varepsilon, L, \delta}^{\Delta t}$ follows immediately from (3.70a-c), (3.13), (3.14) and (3.11b). Similarly to (3.71), the sixth bound in (3.68) then yields that (3.70e) holds for $\widehat{\psi}_{\varepsilon, L, \delta}^{\Delta t, \pm}$. Finally, the desired results $(3.70 \mathrm{f}, \mathrm{g})$ follow immediately from $(3.70 \mathrm{e}),(3.20),(2.3 \mathrm{a})$ and (3.15).

Similarly to (3.72), we have, for any $r \in[2, \infty)$ if $d=2$ or any $r \in[2,6]$ if $d=3$, that

$\begin{array}{rlrl}\|\eta\|_{L^{\frac{2}{\theta}}\left(0, T ; L^{r}(\Omega)\right)} & \leq C\|\eta\|_{L^{2}\left(0, T ; H^{1}(\Omega)\right)} & \text { if } \eta \in L^{\infty}\left(0, T ; L^{2}(\Omega)\right), \quad(3.74 \mathrm{a}) \\ \|\widehat{\varphi}\|_{L^{\frac{2}{\theta}\left(0, T ; L^{r}\left(\Omega ; L_{M}^{2}(D)\right)\right)}} \leq C\|\widehat{\varphi}\|_{L^{2}\left(0, T ; H^{1}\left(\Omega ; L_{M}^{2}(D)\right)\right)} & \text { if } \widehat{\varphi} \in L^{\infty}\left(0, T ; L^{2}\left(\Omega ; L_{M}^{2}(D)\right)\right) ;\end{array}$

where $\theta=d\left(\frac{1}{2}-\frac{1}{r}\right) \in[0,1]$. It follows from (3.69a-d), (3.70g), (3.29), (3.74a), (3.2) and (3.26) that we may pass to the limit, $\Delta t \rightarrow 0_{+}$, in (3.65) to obtain that $u_{\varepsilon, L, \delta} \in$ $L^{\infty}\left(0, T ; L_{\sim}^{2}(\Omega)\right) \cap L^{2}(0, T ; V) \cap W^{1, \frac{4}{d}}\left(0, T ; V_{\sim}^{\prime}\right)$ and $\underset{\sim}{C}\left(M \widehat{\psi}_{\varepsilon, L, \delta}\right) \in L^{\infty}\left(0, T ; \widetilde{\sim}^{2}(\Omega)\right)$ satisfy (3.21a). It also follows from $(3.22)$ that $u_{\varepsilon, L, \delta}(\cdot, 0)=u_{0}(\cdot)$ in the required sense, recall Remark 3.1.

It follows from $(3.70 \mathrm{a}-\mathrm{f}),(3.69 \mathrm{~b}, \mathrm{~d}),(3.74 \mathrm{~b})$ and $(3.8)$ that we may pass to the limit $\Delta t \rightarrow 0_{+}$in $(3.66)$ to obtain that $\widehat{\psi}_{\varepsilon, L, \delta} \in L^{\infty}\left(0, T ; L_{M}^{2}(\Omega \times D)\right) \cap L^{2}(0, T ; \widehat{X}) \cap$ $H^{1}\left(0, T ; \widehat{X}^{\prime}\right)$ and $u_{\varepsilon, L, \delta} \in L^{2}(0, T ; \underset{\sim}{V})$ satisfy $(3.21 \mathrm{~b})$.

Hence we have proved existence of a global weak solution to $\left(\mathrm{P}_{\varepsilon, L, \delta}\right),(3.21 \mathrm{a}, \mathrm{b})$. Moreover, it follows from (3.67), (3.68), (3.69a-c) and (3.70a-g) that

$$
\sup _{t \in(0, T)}\left[\int_{\Omega}\left|u_{\varepsilon, L, \delta}\right|^{2} \mathrm{~d} x\right]+\nu \int_{0}^{T} \int_{\Omega}\left|\underset{\sim}{\nabla} x \underset{\sim}{u_{\varepsilon, L, \delta}}\right|^{2} \mathrm{~d} \underset{\sim}{x} \mathrm{~d} t \leq C(T),
$$




$$
\begin{aligned}
& \sup _{t \in(0, T)}\left[\int_{\Omega \times D} M\left|\widehat{\psi}_{\varepsilon, L, \delta}\right|^{2} \underset{\sim}{\mathrm{d} q} \mathrm{~d} x\right]+\frac{1}{\delta} \sup _{t \in(0, T)}\left[\int_{\Omega \times D} M\left[\widehat{\psi}_{\varepsilon, L, \delta}\right]_{-}^{2} \underset{\sim}{\mathrm{d} q} \mathrm{~d} x\right] \\
& +\int_{0}^{T} \int_{\Omega \times D} M\left[\frac{1}{\lambda}\left|\underset{\sim}{\nabla_{q}} \widehat{\psi}_{\varepsilon, L, \delta}\right|^{2}+\varepsilon\left|{\underset{\sim}{x}}_{x} \widehat{\psi}_{\varepsilon, L, \delta}\right|^{2}\right] \underset{\sim}{\mathrm{d} q \underset{\sim}{\mathrm{d}} x \mathrm{~d} t} \\
& +\sup _{t \in(0, T)}\left[\int_{\Omega}\left|C\left(M \widehat{\psi}_{\varepsilon, L, \delta}\right)\right|^{2} \mathrm{~d} x\right] \\
& +\int_{0}^{T}\left[\left\|\underset{\sim}{S} \frac{\partial u_{\varepsilon, L, \delta}}{\partial t}\right\|_{H^{1}(\Omega)}^{\frac{4}{d}}+\left\|\mathcal{G} \frac{\partial \widehat{\psi}_{\varepsilon, L, \delta}}{\partial t}\right\|_{\widehat{X}}^{\frac{4}{d}}\right] \mathrm{d} t \leq C(L, T) .
\end{aligned}
$$

Remark 3.2. Since the test functions in $\underset{\sim}{V}$ are divergence-free, the pressure has been eliminated in $(3.21 \mathrm{a}, \mathrm{b})$; it can be recovered in a very weak sense following the same procedure as for the incompressible Navier-Stokes equations discussed on p. 208 in $\operatorname{Temam}^{35}$; i.e., one obtains that $\int_{0}^{t} p_{\varepsilon, L, \delta}(\cdot, s) \mathrm{d} s \in C\left([0, T] ; L^{2}(\Omega)\right)$.

\subsection{Existence for $\left(P_{\varepsilon, L}\right)$}

As the bounds $(3.75 \mathrm{a}, \mathrm{b})$ are independent of the parameter $\delta$, it follows immediately, similarly to $(3.69 \mathrm{a}-\mathrm{d}),(3.70 \mathrm{a}-\mathrm{g})$, and $(3.75 \mathrm{a}, \mathrm{b})$, that the following lemma holds.

Lemma 3.3. There exists a subsequence of $\left\{{\underset{u}{\varepsilon, L, \delta}}_{\varepsilon}, \widehat{\psi}_{\varepsilon, L, \delta}\right\}_{\delta>0}$, and functions ${\underset{\sim}{\tau, L}}_{\varepsilon,} \in$ $L^{\infty}\left(0, T ; L_{\tilde{X}}^{2}(\Omega)\right) \cap L^{2}(0, T ; \underset{\sim}{V}) \cap W^{1, \frac{4}{d}}\left(0, T ; V_{\sim}^{\prime}\right)$ and $\widehat{\psi}_{\varepsilon, L} \in L^{\infty}\left(0, T ; L_{M}^{2}(\Omega \times D)\right) \cap$ $L^{2}(0, T ; \widetilde{X}) \cap H^{1}\left(0, T ; \widehat{X}^{\prime}\right)$, with $\widehat{\psi}_{\varepsilon, L} \geq 0$ a.e. in $\Omega \times D \times(0, T)$, such that, as $\delta \rightarrow 0_{+}$,

$$
\begin{aligned}
& {\underset{\sim}{u}, L, \delta}_{\varepsilon} \rightarrow u_{\varepsilon, L} \quad \text { weak }{ }^{*} \text { in } L^{\infty}\left(0, T ; L_{\sim}^{2}(\Omega)\right), \\
& {\underset{\sim}{\varepsilon, L, \delta}}_{\sim} \rightarrow{\underset{\sim}{\varepsilon, L}}_{\varepsilon} \quad \text { weakly in } L^{2}(0, T ; V) \text {, } \\
& \underset{\sim}{S} \frac{\partial u_{\varepsilon, L, \delta}}{\partial t} \rightarrow \underset{\sim}{S} \frac{\partial u_{\varepsilon, L}}{\partial t} \quad \text { weakly in } L^{\frac{4}{d}}(0, T ; \underset{\sim}{V}) \\
& {\underset{\sim}{u, L, \delta}}_{\varepsilon} \rightarrow{\underset{\sim}{u}, L}_{\varepsilon} \quad \text { strongly in } L^{2}\left(0, T ; L_{\sim}^{r}(\Omega)\right) \text {, }
\end{aligned}
$$


where $r \in[1, \infty)$ if $d=2$ and $r \in[1,6)$ if $d=3$; and

$$
\begin{aligned}
& M^{\frac{1}{2}} \widehat{\psi}_{\varepsilon, L, \delta} \rightarrow M^{\frac{1}{2}} \widehat{\psi}_{\varepsilon, L} \quad \text { weak }^{*} \text { in } L^{\infty}\left(0, T ; L^{2}(\Omega \times D)\right), \\
& M^{\frac{1}{2}}{\underset{\sim}{q}}_{q} \widehat{\psi}_{\varepsilon, L, \delta} \rightarrow M^{\frac{1}{2}}{\underset{\sim}{q}}_{q} \widehat{\psi}_{\varepsilon, L} \quad \text { weakly in } L^{2}\left(0, T ; L_{\sim}^{2}(\Omega \times D)\right), \\
& M^{\frac{1}{2}} \underset{\sim}{\nabla_{x}} \widehat{\psi}_{\varepsilon, L, \delta} \rightarrow M^{\frac{1}{2}} \underset{\sim}{\nabla_{x}} \widehat{\psi}_{\varepsilon, L} \quad \text { weakly in } L^{2}\left(0, T ; \underset{\sim}{\left.L^{2}(\Omega \times D)\right),}\right. \\
& \mathcal{G} \frac{\partial \widehat{\psi}_{\varepsilon, L, \delta}}{\partial t} \rightarrow \mathcal{G} \frac{\partial \widehat{\psi}_{\varepsilon, L}}{\partial t} \quad \text { weakly in } L^{\frac{4}{d}}(0, T ; \widehat{X}), \\
& M^{\frac{1}{2}} \widehat{\psi}_{\varepsilon, L, \delta} \rightarrow M^{\frac{1}{2}} \widehat{\psi}_{\varepsilon, L} \quad \text { strongly in } L^{2}\left(0, T ; L^{2}(\Omega \times D)\right), \\
& M^{\frac{1}{2}} \beta_{\delta}^{L}\left(\widehat{\psi}_{\varepsilon, L, \delta}\right) \rightarrow M^{\frac{1}{2}} \beta^{L}\left(\widehat{\psi}_{\varepsilon, L}\right) \quad \text { strongly in } L^{\infty}\left(0, T ; L^{\infty}(\Omega \times D)\right) \text {, } \\
& \underset{\approx}{C}\left(M \widehat{\psi}_{\varepsilon, L, \delta}\right) \rightarrow \underset{\approx}{C}\left(M \widehat{\psi}_{\varepsilon, L}\right) \quad \text { strongly in } L^{2}(0, T ; \underset{\approx}{\underset{\sim}{2}(\Omega)) .}
\end{aligned}
$$

In addition, we have that

$$
\begin{aligned}
& \sup _{t \in(0, T)}\left[\int_{\Omega}\left|u_{\varepsilon, L}\right|^{2} \mathrm{~d} x\right]+\nu \int_{0}^{T} \int_{\Omega}\left|\underset{\approx}{\nabla_{x}} \underset{\sim}{u_{\varepsilon, L}}\right|^{2} \mathrm{~d} x \mathrm{\sim} \mathrm{d} t \leq C(T), \\
& \sup _{t \in(0, T)}\left[\int_{\Omega \times D} M^{\frac{1}{2}}\left|\widehat{\psi}_{\varepsilon, L}\right|^{2} \mathrm{~d} q \underset{\sim}{\mathrm{d} x}\right]+\sup _{t \in(0, T)}\left[\int_{\Omega}\left|C\left(M \widehat{\psi}_{\varepsilon, L}\right)\right|^{2} \mathrm{~d} x\right] \\
& +\int_{0}^{T} \int_{\Omega \times D} M\left[\frac{1}{\lambda}\left|\underset{\sim}{\nabla_{q}} \widehat{\psi}_{\varepsilon, L}\right|^{2}+\varepsilon\left|\underset{\sim}{\nabla_{x}} \widehat{\psi}_{\varepsilon, L}\right|^{2}\right] \underset{\sim}{\mathrm{d} q} \underset{\sim}{\mathrm{d} x} \mathrm{~d} t \\
& +\int_{0}^{T}\left[\left\|\underset{\sim}{S} \frac{\partial u_{\varepsilon, L}}{\partial t}\right\|_{H^{1}(\Omega)}^{\frac{4}{d}}+\left\|\mathcal{G} \frac{\partial \widehat{\psi}_{\varepsilon, L}}{\partial t}\right\|_{\widehat{X}}^{\frac{4}{d}}\right] \mathrm{d} t \leq C(L, T) .
\end{aligned}
$$

In particular, the non-negativity of $\widehat{\psi}_{\varepsilon, L}$ in the above lemma follows from the second bound in $(3.75 \mathrm{~b})$. Therefore we can then pass to limit $\delta \rightarrow 0_{+}$in $\left(\mathrm{P}_{\varepsilon, L, \delta}\right)$ to obtain global existence of a weak solution to the following problem for given $\varepsilon \in(0,1]$ and $L>1$ :

$\left(\mathrm{P}_{\varepsilon, L}\right)$ Find $\underset{\sim}{u_{\varepsilon, L}} \in L^{\infty}\left(0, T ; L^{2}(\Omega)\right) \cap L^{2}(0, T ; \underset{\sim}{V}) \cap W^{1, \frac{4}{d}}\left(0, T ; V^{\prime}\right)$ and $\widehat{\psi}_{\varepsilon, L} \in L^{\infty}\left(0, T ; L_{M}^{2}(\Omega \times D)\right) \cap L^{2}(0, T ; \widehat{X}) \cap H^{1}\left(0, T ; \widehat{X}^{\prime}\right)$, with $\underset{\approx}{C}\left(M \widehat{\psi}_{\varepsilon, L}\right) \in$ 
$L^{\infty}\left(0, T ; \underset{\sim}{L^{2}}(\Omega)\right)$, such that $u_{\varepsilon, L}(\cdot, 0)={\underset{\sim}{u}}_{0}(\cdot), \widehat{\psi}_{\varepsilon, L}(\cdot, 0)=\widehat{\psi}_{0}(\cdot)$ and

$$
\begin{aligned}
& \int_{0}^{T}\left\langle\frac{\partial u_{\varepsilon, L}}{\partial t}, \underset{\sim}{w}\right\rangle_{V} \mathrm{~d} t \\
& +\int_{0}^{T} \int_{\Omega}\left[\left[\left(\underset{\sim}{u_{\varepsilon}, L} \cdot \underset{\sim}{\nabla} x\right) \underset{\sim}{u_{\varepsilon}} u_{L}\right] \cdot \underset{\sim}{w}+\nu \underset{\sim}{\underset{\sim}{\nabla}} \underset{\sim}{u_{\varepsilon, L}}: \underset{\sim}{\underset{\sim}{\nabla}} \underset{\sim}{w}\right] \underset{\sim}{\mathrm{d} x} \mathrm{~d} t \\
& =\int_{0}^{T}\langle\underset{\sim}{f f} \underset{\sim}{w}\rangle_{V} \mathrm{~d} t-k \mu \int_{0}^{T} \int_{\Omega} \underset{\approx}{C}\left(M \widehat{\psi}_{\varepsilon}, L\right): \underset{\approx}{\nabla_{x}} \underset{\sim}{w} \mathrm{~d} x \mathrm{\sim} d \quad \forall \underset{\sim}{w} \in L^{\frac{4}{4-d}}(0, T ; \underset{\sim}{V}), \\
& \int_{0}^{T}\left\langle M \frac{\partial \widehat{\psi}_{\varepsilon, L}}{\partial t}, \widehat{\varphi}\right\rangle_{\widehat{X}} \mathrm{~d} t \\
& \left.\left.+\int_{0}^{T} \int_{\Omega \times D} M\left[\frac{1}{2 \lambda} \underset{\sim}{\nabla} q \widehat{\psi}_{\varepsilon, L}-\underset{\sim}{\sigma} \underset{\sim}{\left(u_{\varepsilon, L}\right.}\right) \underset{\sim}{q}\right] \beta^{L}\left(\widehat{\psi}_{\varepsilon, L}\right)\right] \cdot \underset{\sim}{\nabla_{q}} \widehat{\varphi} \underset{\sim}{\widehat{d} q} \mathrm{~d} x \mathrm{~d} t
\end{aligned}
$$

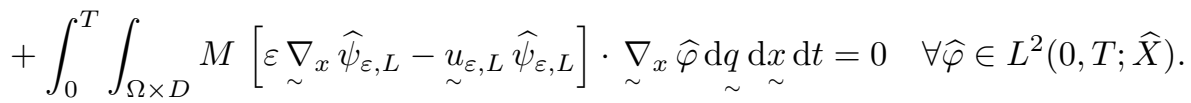

Remark 3.3. Although we have introduced $x$-diffusion and a cut-off above to $\widehat{\psi}=\psi / M$ in the drag term in the Fokker-Planck equation through the parameters $\varepsilon \in(0,1]$ and $L>1$ in the model $\left(\mathrm{P}_{\varepsilon, L}\right)$ compared to the standard polymer model, (P); we wish to stress that the bounds on $u_{\varepsilon, L}$, the variable of real physical interest, in (3.78a) are independent of these parameters $\varepsilon$ and $L$.

Remark 3.4. We also note that, for any $s \in(0, T)$ and $\Delta t$ sufficiently small such that $0<\Delta t<s$, we can choose $\widehat{\varphi}(\underset{\sim}{x}, \underset{\sim}{q}, t)=\frac{1}{\Delta t}\left\{[s-t]_{+}-[s-\Delta t-t]_{+}\right\}$in $\left(\mathrm{P}_{\varepsilon, L}\right)$ to yield that

$$
\frac{1}{\Delta t} \int_{s-\Delta t}^{s} \int_{\Omega \times D} M \widehat{\psi}_{\varepsilon, L}(\underset{\sim}{x}, \underset{\sim}{q}, t) \mathrm{d} \underset{\sim}{q} \mathrm{~d} x \underset{\sim}{\mathrm{d}} t=\int_{\Omega \times D} M \widehat{\psi}_{0}(\underset{\sim}{x} \underset{\sim}{q}) \mathrm{d} \underset{\sim}{q} \mathrm{~d} x .
$$

Passing to the limit $\Delta t \rightarrow 0_{+}$, we deduce that

$$
\int_{\Omega \times D} M \widehat{\psi}_{\varepsilon, L}(\underset{\sim}{x}, \underset{\sim}{q}, s) \mathrm{d} \underset{\sim}{q} \mathrm{~d} x=\int_{\Omega \times D} M \psi_{0}(\underset{\sim}{x} \underset{\sim}{q}) \mathrm{d} q \underset{\sim}{\mathrm{d} x} \quad \forall s \in(0, T) .
$$

An identical statement can be made about $\widehat{\psi}_{\varepsilon, L, \delta}$ in $\left(\mathrm{P}_{\varepsilon, L, \delta}\right)$.

Remark 3.5. In the case of a corotational model (i.e. with $\underset{\widetilde{\sim}}{\sigma} \underset{\sim}{v})=\nabla_{x} \underset{\sim}{v}$ replaced by $\sigma_{\text {corot }}(\underset{\sim}{v}):=\frac{1}{2}\left({\underset{\sim}{x}}_{x} \underset{\sim}{v}-\left({\underset{\sim}{\nabla}}_{x} \underset{\sim}{v}\right)^{\top}\right)$ in the drag term in the Fokker-Planck equation), the right-hand sides in the estimates (3.55) and (3.56) become independent of $L$, as one can exploit additional cancellations due to the skew-symmetry of $\underset{\sim}{\sigma} \operatorname{corot}(\underset{\sim}{v})$. Hence, (3.59) is then also independent of $L$. This raises the question whether in the case of a corotational model one can pass to the limit $L \rightarrow \infty$ to recover the 
Fokker-Planck equation, without cut-off. The answer to this question is positive, however some modifications are required in the arguments above in order to show this. For the sake of brevity, we omit the details and only highlight the key changes needed.

In our discussion above, because of the cut-off, we control also the time derivative of $\widehat{\psi}_{\varepsilon, L, \delta}$; without cut-off this does not appear to be possible. In addition, one should avoid (3.62) as the right-hand side of this inequality remains $L$-dependent regardless of whether or not the drag term is corotational. It is possible to get around these technical difficulties by proceeding as in Barrett and Süli ${ }^{5}$. Firstly, the time derivative has to be transferred from $\widehat{\psi}_{\varepsilon, L, \delta}$ to the (time-dependent) test function in the weak formulation of the Fokker-Planck equation. Secondly, as we will no longer have strong convergence of a subsequence of $\left\{\widehat{\psi}_{\varepsilon, L, \delta}\right\}_{\delta>0}$ to $\widehat{\psi}_{\varepsilon, L}$ as $\delta \rightarrow 0_{+}$, and of $\left\{\widehat{\psi}_{\varepsilon, L}\right\}_{L>1}$ to $\widehat{\psi}_{\varepsilon}$ as $L \rightarrow \infty$, the drag term has to be rewritten using the fact that, for all $\underset{\sim}{v} \in \underset{\sim}{H} H_{0}^{1}(\Omega)$ and $\underset{\sim}{\hat{\varphi}} \in \underset{\sim}{H^{1}}\left(\Omega ; L_{M}^{2}(D)\right)$,

$$
\int_{\Omega \times D} M[\underset{\sim}{\sigma} \operatorname{corot}(\underset{\sim}{v}) \underset{\sim}{q}] \cdot \underset{\sim}{\widehat{\varphi}} \mathrm{d} \underset{\sim}{q} \mathrm{~d} x=\frac{1}{2} \int_{\Omega \times D} M\left[(\underset{\sim}{v} \cdot \underset{\sim}{q})(\underset{\sim}{\nabla} \cdot \underset{\sim}{\widehat{\varphi}})-\left[\left(\nabla_{x} \underset{\sim}{\widehat{\varphi}}\right) \underset{\sim}{q}\right] \cdot \underset{\sim}{v}\right] \mathrm{d} q \underset{\sim}{\mathrm{d} x} .
$$

One can then pass to the simultaneous limits $\delta \rightarrow 0_{+}$and $L \rightarrow \infty$ in a very similar manner as we did in the final section of Barrett and Süli ${ }^{5}$.

\section{Appendix A. Compact embedding of Maxwellian-weighted spaces}

Let us suppose that $D$ is a bounded open ball in $\mathbb{R}^{d}$ centred at $\underset{\sim}{0} \in \mathbb{R}^{d}$, and let $U$ and $M$ be as in section 1. Our aim is to prove that the embedding of the Maxwellianweighted Sobolev space $H_{M}^{1}(\Omega \times D)$ into the Maxwellian-weighted Lebesgue space $L_{M}^{2}(\Omega \times D)$ is compact. The proof proceeds in three steps.

\section{A.1. Step 1: Compact embedding of $H_{M}^{1}(D)$ into $L_{M}^{2}(D)$, completeness and separability}

We may suppose, with no loss of generality, that $D=B\left(\underset{\sim}{0}, b^{\frac{1}{2}}\right)$, with $b>0$, as in the case of the FENE model, whereby $\mathcal{O}=\left[0, \frac{b}{2}\right)$. As in section 1 , we shall assume that $U \in C^{\infty}\left(\mathcal{O} ; \mathbb{R}_{\geq 0}\right), U(0)=0, U$ is monotonic increasing with $\lim _{s \rightarrow(b / 2)-} U(s)=$ $+\infty$, and $U$ and the associated Maxwellian $M$ satisfy $(1.3 \mathrm{a}, \mathrm{b})$ with $\gamma \geq 1$. Elsewhere in the paper we require $\gamma>1$ (cf. (3.5)).

Let

$$
f(r):=\lambda \mathrm{e}^{-U\left(\frac{1}{2}\left(b^{\frac{1}{2}}-r\right)^{2}\right)} \quad \forall r \in\left(0, b^{\frac{1}{2}}\right],
$$

where $\lambda \in \mathbb{R}_{>0}$. Clearly, $\lim _{r \rightarrow 0_{+}} f(r)=0, \lim _{r \rightarrow 0_{+}} f^{\prime}(r)=0, f$ is positive and increasing on $\left(0, b^{\frac{1}{2}}\right], f \in C^{1}\left[0, b^{\frac{1}{2}}\right]$, and

$$
M(\underset{\sim}{q})=f\left(b^{\frac{1}{2}}-|\underset{\sim}{q}|\right) \quad \forall \underset{\sim}{q}:|\underset{\sim}{q}|<b^{\frac{1}{2}} .
$$

With this choice of $D$ and $f$, the compactness of the embedding of the Maxwellian-weighted Sobolev space $H_{M}^{1}(D)$ into the Maxwellian-weighted Lebesgue 


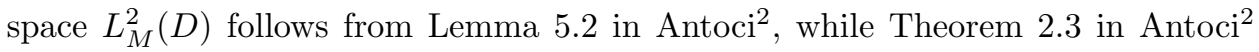
implies, with $p=2$, that $H_{M}^{1}(D)$ and $L_{M}^{2}(D)$ are Hilbert spaces.

As a matter of fact, $H_{M}^{1}(D)$ and $L_{M}^{2}(D)$ are separable Hilbert spaces. This, as we shall prove below, follows on noting that $C^{1}(\bar{D})$ is a separable Banach space (e.g. the set $\mathcal{P}$ of all polynomials with rational coefficients is a countable dense subset of $C^{1}(\bar{D})$ ) and that, by Theorem 3.2.2(c) in Triebel $^{36}, C^{\infty}(\bar{D})$ is dense in both $H_{M}^{1}(D)$ and $L_{M}^{2}(D)$.

Indeed, given $v \in H_{M}^{1}(D)$ and any $\varepsilon>0$ there exists $\varphi \in C^{\infty}(\bar{D})$ such that

$$
\|v-\varphi\|_{H_{M}^{1}(D)}<\frac{1}{2} \varepsilon .
$$

Since $C^{1}(\bar{D})$ is separable, there exists a countable dense set $\mathcal{P} \subset C^{1}(\bar{D})$; hence, given $\varepsilon>0$ there exists $p \in \mathcal{P}$ such that

$$
\|\varphi-p\|_{C^{1}(\bar{D})}<\frac{1}{2}\left(\int_{D} M(\underset{\sim}{q}) \underset{\sim}{q}\right)^{-1 / 2} \varepsilon .
$$

Clearly, $C^{1}(\bar{D}) \subset H_{M}^{1}(D)$ and therefore $\mathcal{P} \subset H_{M}^{1}(D)$. Thus,

$$
\begin{aligned}
\|v-p\|_{H_{M}^{1}(D)} & \leq\|v-\varphi\|_{H_{M}^{1}(D)}+\|\varphi-p\|_{H_{M}^{1}(D)} \\
& <\frac{1}{2} \varepsilon+\|\varphi-p\|_{C^{1}(\bar{D})}\left(\int_{D} M(q) \underset{\sim}{\mathrm{d} q}\right)^{1 / 2}<\varepsilon .
\end{aligned}
$$

This shows that the countable set $\mathcal{P} \subset H_{M}^{1}(D)$ is dense in $H_{M}^{1}(D)$. Therefore $H_{M}^{1}(D)$ is separable. By an identical argument, $L_{M}^{2}(D)$ is separable.

\section{A.2. Step 2: Isometric isomorphisms}

Let $\Omega$ be a bounded open Lipschitz domain in $\mathbb{R}^{d}$. We now show the isometric isomorphism of the following pairs of spaces, respectively: $L_{M}^{2}(\Omega \times D)$ and $L^{2}\left(\Omega ; L_{M}^{2}(D)\right) ; H_{M}^{0,1}(\Omega \times D)$ and $L^{2}\left(\Omega ; H_{M}^{1}(D)\right) ; H_{M}^{1,0}(\Omega \times D)$ and $H^{1}\left(\Omega ; L_{M}^{2}(D)\right)$. For a precise definition of $H_{M}^{0,1}(\Omega \times D)$ and $H_{M}^{1,0}(\Omega \times D)$, see below.

\section{A.2.1. Isometric isomorphism of $L_{M}^{2}(\Omega \times D)$ and $L^{2}\left(\Omega ; L_{M}^{2}(D)\right)$}

Let

$$
L^{2}\left(\Omega ; L_{M}^{2}(D)\right):=\left\{v \in \mathcal{M}_{w}\left(\Omega, L_{M}^{2}(D)\right): \int_{\Omega}\|v(\underset{\sim}{x})\|_{L_{M}^{2}(D)}^{2} \mathrm{~d} \underset{\sim}{x}<\infty\right\},
$$

where

$$
\mathcal{M}_{w}\left(\Omega, L_{M}^{2}(D)\right):=\left\{v: \Omega \rightarrow L_{M}^{2}(D): v \text { is weakly measurable on } \Omega\right\} .
$$

Let $\left\{\varphi_{j}\right\}_{j=1}^{\infty}$ be a complete orthonormal system in the (separable) Hilbert space $L_{M}^{2}(D)$ with respect to the inner product $(\cdot, \cdot)$ of $L_{M}^{2}(D)$. For $v \in L^{2}\left(\Omega ; L_{M}^{2}(D)\right)$, we define the function

$$
V_{N}(\underset{\sim}{x} \underset{\sim}{q}):=\sum_{j=1}^{N}\left(v(\underset{\sim}{x}), \varphi_{j}\right) \varphi_{j}(\underset{\sim}{q}) .
$$


As $v$ is weakly measurable on $\Omega$, each of the functions $\underset{\sim}{x} \mapsto\left(v(\underset{\sim}{x}), \varphi_{j}\right), j=1,2, \ldots$, is measurable on $\Omega$; therefore $(\underset{\sim}{x}, q) \mapsto\left(v(\underset{\sim}{x}), \varphi_{j}\right)$ is measurable on $\Omega \times D$ for all $j=1,2, \ldots$ Similarly, $\underset{\sim}{q} \mapsto \varphi_{j}(\underset{\sim}{q})^{\sim}$ is measurable on $D$ for each $j=1,2, \ldots$, and therefore $(\underset{\sim}{x}, q) \mapsto \varphi_{j}(\underset{\sim}{q})$ is measurable on $\Omega \times D$. Hence, also $V_{N}$ is a measurable function on $\tilde{\Omega} \times D$. Now,

$$
\left.\left|V_{N}(\underset{\sim}{x} \underset{\sim}{q})\right|^{2}=\sum_{j=1}^{N} \sum_{k=1}^{N}\left(v(\underset{\sim}{x}), \varphi_{j}\right)\left(v(\underset{\sim}{x}), \varphi_{k}\right) \varphi_{j} \underset{\sim}{q}\right) \varphi_{k}(\underset{\sim}{q}) .
$$

By the Cauchy-Schwarz inequality $M \varphi_{j} \varphi_{k}=M^{\frac{1}{2}} \varphi_{j} \cdot M^{\frac{1}{2}} \varphi_{k} \in L^{1}(D)$ for all $j, k \geq$ 1 ; hence also $M(\cdot)\left|V_{N}(\underset{\sim}{x}, \cdot)\right|^{2} \in L^{1}(D)$ for a.e. $\underset{\sim}{x} \in \Omega$. Thus, by the orthonormality of the $\varphi_{j}, j=1,2, \ldots$, in $L_{M}^{2}(D)$,

$$
\int_{D} M(\underset{\sim}{q})\left|V_{N}(\underset{\sim}{x} \underset{\sim}{q})\right|^{2} \mathrm{~d} \underset{\sim}{q}=\sum_{j=1}^{N}\left|\left(v(\underset{\sim}{x}), \varphi_{j}\right)\right|^{2}, \quad \text { a.e. } \underset{\sim}{x} \in \Omega .
$$

By Bessel's inequality in $L_{M}^{2}(D)$, the right-hand side of this last equality is bounded by $\|v(\underset{\sim}{x})\|_{L_{M}^{2}(D)}^{2}$ for a.e. $\underset{\sim}{x} \in \Omega$, and, by hypothesis, $\underset{\sim}{x} \mapsto v(\underset{\sim}{x}) \in L^{2}(\Omega)$; therefore, by Fubini's theorem, $M\left|V_{N}\right|^{2} \in L^{1}(\Omega \times D)$. Upon integrating both sides over $\Omega$, and using Fubini's theorem on the left-hand side to write the multiple integral over $\Omega$ and $D$ as an integral over $\Omega \times D$, we have

$$
\left\|V_{N}\right\|_{L_{M}^{2}(\Omega \times D)}^{2}:=\int_{\Omega \times D} M(\underset{\sim}{q})\left|V_{N}(\underset{\sim}{x} \underset{\sim}{q})\right|^{2} \mathrm{~d} \underset{\sim}{q} \mathrm{~d} \underset{\sim}{x}=\sum_{j=1}^{N} \int_{\Omega}\left|\left(v(\underset{\sim}{x}), \varphi_{j}\right)\right|^{2} \mathrm{~d} \underset{\sim}{x} .
$$

Now, let

$$
\left.y_{N}(\underset{\sim}{x}):=\sum_{j=1}^{N} \mid(v \underset{\sim}{x}), \varphi_{j}\right)\left.\right|^{2}, \quad \underset{\sim}{x} \in \Omega .
$$

The sequence $\left\{y_{N}(\underset{\sim}{x})\right\}_{N=1}^{\infty}$ is monotonic increasing for almost all $\underset{\sim}{x} \in \Omega$; also, according to Bessel's inequality in $L_{M}^{2}(D)$ we have that

$$
0 \leq y_{N}(\underset{\sim}{x}) \leq\|v(\underset{\sim}{x})\|_{L_{M}^{2}(D)}^{2} \quad \forall N \geq 1, \quad \text { a.e. } \underset{\sim}{x} \in \Omega .
$$

Thus $\left\{y_{N}(\underset{\sim}{x})\right\}_{N=1}^{\infty}$ is a bounded sequence of real numbers, for a.e. $\underset{\sim}{x} \in \Omega$. Therefore, the sequence $\left\{y_{N}(\underset{\sim}{x})\right\}_{N=1}^{\infty}$ converges in $\mathbb{R}$ for a.e. $\underset{\sim}{x} \in \mathbb{R}$, with

$$
\left.y(\underset{\sim}{x})=\lim _{N \rightarrow \infty} y_{N}(\underset{\sim}{x})=\sum_{j=1}^{\infty} \mid(v \underset{\sim}{x}), \varphi_{j}\right)\left.\right|^{2}, \quad \text { a.e. } \underset{\sim}{x} \in \Omega .
$$

By the monotone convergence theorem,

$$
\begin{aligned}
\lim _{N \rightarrow \infty} \sum_{j=1}^{N} \int_{\Omega}\left|\left(v(\underset{\sim}{x}), \varphi_{j}\right)\right|^{2} \mathrm{~d} \underset{\sim}{x} & =\lim _{N \rightarrow \infty} \int_{\Omega} y_{N}(\underset{\sim}{x}) \mathrm{d} \underset{\sim}{x} \\
& =\int_{\Omega} y(\underset{\sim}{x}) \mathrm{d} \underset{\sim}{x}=\int_{\Omega} \sum_{j=1}^{\infty}\left|\left(v(\underset{\sim}{x}), \varphi_{j}\right)\right|^{2} \mathrm{~d} \underset{\sim}{x} .
\end{aligned}
$$


This implies that

$$
\left\{\sum_{j=1}^{N} \int_{\Omega}\left|\left(v(x), \varphi_{j}\right)\right|^{2} \mathrm{~d} x\right\}_{N=1}^{\infty}
$$

is a convergent sequence of real numbers. Hence, it is also a Cauchy sequence in $\mathbb{R}$.

Since, for any $N>L \geq 1$,

$$
\int_{\Omega \times D}\left|V_{N}(\underset{\sim}{x} \underset{\sim}{q})-V_{L}(\underset{\sim}{x} \underset{\sim}{q})\right|^{2} \mathrm{~d} \underset{\sim}{q} \mathrm{~d} x=\sum_{j=L+1}^{N} \int_{D}\left|\left(v(\underset{\sim}{x}), \varphi_{j}\right)\right|^{2} \mathrm{~d} x,
$$

it follows that $\left\{V_{N}\right\}_{N=1}^{\infty}$ is a Cauchy sequence in $L_{M}^{2}(\Omega \times D)$. Since $L_{M}^{2}(\Omega \times D)$ is a Hilbert space, there exists a unique $V \in L_{M}^{2}(\Omega \times D)$ such that

$$
V=\lim _{N \rightarrow \infty} V_{N} \quad \text { in } L_{M}^{2}(\Omega \times D) .
$$

Thus we have shown that the mapping

$$
\mathcal{I}: v \in L^{2}\left(\Omega, L_{M}^{2}(D)\right) \mapsto V:=\sum_{j=1}^{\infty}\left(v(\cdot), \varphi_{j}\right) \varphi_{j}(\cdot) \in L_{M}^{2}(\Omega \times D)
$$

is correctly defined. Next, we prove that $\mathcal{I}$ is a bijective isometry, and this will imply that the spaces $L^{2}\left(\Omega ; L_{M}^{2}(D)\right)$ and $L_{M}^{2}(\Omega \times D)$ are isometrically isomorphic.

We begin by showing that $\mathcal{I}$ is injective. As $\mathcal{I}$ is linear it suffices to prove that if $\mathcal{I}(v)=0$ then $v=0$. Indeed, if $\mathcal{I}(v)=0$, then

$$
\sum_{j=1}^{\infty}\left(v(\underset{\sim}{x}), \varphi_{j}\right) \varphi_{j}(\underset{\sim}{q})=0 \quad \text { for a.e. }(\underset{\sim}{x}, \underset{\sim}{q}) \in \Omega \times D .
$$

Since $\left\{\varphi_{j}\right\}_{j=1}^{\infty}$ is an orthonormal system in $L_{M}^{2}(D)$, it follows that $\left(v(x), \varphi_{j}\right)=0$ for a.e. $\underset{\sim}{x} \in \Omega$ and all $j=1,2, \ldots$. The completeness of the orthonormal system $\left\{\varphi_{j}\right\}_{j=1}^{\infty}$ in $L_{M}^{2}(D)$ now implies that $v(\underset{\sim}{x})=0$ in $L_{M}^{2}(D)$ for a.e. $\underset{\sim}{x} \in \Omega$, i.e. $v=0$ in $L^{2}\left(\Omega ; L_{M}^{2}(D)\right)$.

Next we show that $\mathcal{I}$ is surjective. Suppose that $V \in L_{M}^{2}(\Omega \times D)$. Then, by Fubini's theorem, $V(\underset{\sim}{x}, \cdot) \in L_{M}^{2}(D)$ for a.e. $\underset{\sim}{x} \in \Omega$. Since $\left\{\varphi_{j}\right\}_{j=1}^{\infty}$ is a complete orthonormal system in $L_{M}^{2}(D)$, it follows that

$$
V(\underset{\sim}{x}, \cdot)=\sum_{j=1}^{\infty}\left(V(\underset{\sim}{x}, \cdot), \varphi_{j}\right) \varphi_{j}(\cdot) .
$$

On defining $v(\underset{\sim}{x}):=V(\underset{\sim}{x}, \cdot) \in L_{M}^{2}(D)$, we have that $\mathcal{I}(v)=V$. Hence $\mathcal{I}$ is surjective. 
Finally, we show that $\mathcal{I}$ is an isometry. Clearly

$$
\begin{aligned}
\|V\|_{L_{M}^{2}(\Omega \times D)}^{2} & \stackrel{(A .3)}{=} \lim _{N \rightarrow \infty}\left\|V_{N}\right\|_{L_{M}^{2}(\Omega \times D)}^{2} \\
\stackrel{(A .1)}{=} & \lim _{N \rightarrow \infty} \sum_{j=1}^{N} \int_{\Omega}\left|\left(v(\underset{\sim}{x}), \varphi_{j}\right)\right|^{2} \mathrm{~d} \underset{\sim}{x} \\
& \stackrel{(A .2)}{=} \int_{\Omega} \sum_{j=1}^{\infty}\left|\left(v(\underset{\sim}{x}), \varphi_{j}\right)\right|^{2} \mathrm{~d} \underset{\sim}{x} .
\end{aligned}
$$

Applying Parseval's identity in $L_{M}^{2}(D)$ to the infinite series under the last integral sign, we deduce that

$$
\|V\|_{L_{M}^{2}(\Omega \times D)}^{2}=\int_{\Omega}\|v(\underset{\sim}{x})\|_{L_{M}^{2}(D)}^{2} \mathrm{~d} \underset{\sim}{x}=\|v\|_{L^{2}\left(\Omega ; L_{M}^{2}(D)\right)}^{2} .
$$

Thus we have shown that $\|\mathcal{I} v\|_{L_{M}^{2}(\Omega \times D)}=\|v\|_{L^{2}\left(\Omega ; L^{2}(D)\right)}$, whereby $\mathcal{I}$ is an isometry.

\section{A.2.2. Isometric isomorphism of $H_{M}^{0,1}(\Omega \times D)$ and $L^{2}\left(\Omega ; H_{M}^{1}(D)\right)$}

Let us begin by observing that $L_{M}^{2}(\Omega \times D) \subset L_{\text {loc }}^{1}(\Omega \times D)$, and therefore any $V$ in $L_{M}^{2}(\Omega \times D)$ can be considered to be an element of $\mathcal{D}^{\prime}(\Omega \times D)$, the space of $\mathbb{R}$-valued distributions on $\Omega \times D$. Let $\nabla_{q}$ denote the distributional gradient with respect to $\underset{\sim}{q}$, defined on $\mathcal{D}^{\prime}(\Omega \times D)$. We define

$$
H_{M}^{0,1}(\Omega \times D):=\left\{V \in L_{M}^{2}(\Omega \times D): \nabla_{q} V \in L_{M}^{2}(\Omega \times D)\right\} .
$$

A completely identical argument to the one above shows that $H_{M}^{0,1}(\Omega \times D)$ is isometrically isomorphic to $L^{2}\left(\Omega ; H_{M}^{1}(D)\right)$; the only change that is required is to replace $L_{M}^{2}(D)$ by $H_{M}^{1}(D)$ throughout and to take $\left\{\varphi_{j}\right\}_{j=1}^{\infty}$ to be a complete orthonormal system in the inner product $(\cdot, \cdot)$ of the (separable) Hilbert space $H_{M}^{1}(D)$, instead of $L_{M}^{2}(D)$.

\section{A.2.3. Isometric isomorphism of $H_{M}^{1,0}(\Omega \times D)$ and $H^{1}\left(\Omega ; L_{M}^{2}(D)\right)$}

Concerning the isometric isomorphism of $H_{M}^{1,0}(\Omega \times D)$ and $H^{1}\left(\Omega ; L_{M}^{2}(D)\right)$ we proceed as follows. Given $v \in H^{1}\left(\Omega ; L_{M}^{2}(D)\right) \subset L^{2}\left(\Omega ; L_{M}^{2}(D)\right)$, we define, as in the proof of the isometric isomorphism of $L^{2}\left(\Omega ; L_{M}^{2}(D)\right)$ and $L_{M}^{2}(\Omega \times D)$ above, the function

$$
V:(\underset{\sim}{x} \underset{\sim}{q}) \in \Omega \times D \mapsto V(\underset{\sim}{x} \underset{\sim}{q}):=\sum_{j=1}^{\infty}\left(v(\underset{\sim}{x}), \varphi_{j}\right) \varphi_{j}(\underset{\sim}{q}) \in \mathbb{R},
$$

where $\left\{\varphi_{j}\right\}_{j=1}^{\infty}$ is a complete orthonormal system in $L_{M}^{2}(D)$. We showed above that $V \in L_{M}^{2}(\Omega \times D)$, and $\|V\|_{L_{M}^{2}(\Omega \times D)}=\|v\|_{L^{2}\left(\Omega ; L_{M}^{2}(D)\right)}$.

Let $\nabla_{x}$ denote the distributional gradient with respect to $\underset{\sim}{x}$, defined on $\mathcal{D}^{\prime}(\Omega \times$ $D)$, and let $D_{x}$ denote the distributional gradient, defined on $\mathcal{D}^{\prime}\left(\Omega ; L_{M}^{2}(D)\right)$, the 
space of $L_{M}^{2}(D)$-valued distributions on $\Omega$. Applying $\underset{\sim}{\nabla_{x}}$ to

$$
V=\sum_{j=1}^{\infty}\left(v, \varphi_{j}\right) \varphi_{j} \quad \text { in } \mathcal{D}^{\prime}(\Omega \times D)
$$

and noting that

$$
\nabla_{x} V=\sum_{j=1}^{\infty}\left(D_{x} v, \varphi_{j}\right) \varphi_{j}
$$

it follows from the isometric isomorphism of $L_{M}^{2}(\Omega \times D)$ and $L^{2}\left(\Omega ; L_{M}^{2}(D)\right)$ that

$$
\begin{aligned}
\|V\|_{H_{M}^{1,0}(\Omega \times D)}^{2} & =\|V\|_{L_{M}^{2}(\Omega \times D)}^{2}+\left\|\nabla_{x} V\right\|_{L_{M}^{2}(\Omega \times D)}^{2} \\
& =\|v\|_{L_{M}^{2}\left(\Omega ; L_{M}^{2}(D)\right)}^{2}+\left\|D_{x} v\right\|_{L^{2}\left(\Omega ; L_{M}^{2}(D)\right)}^{2} \\
& =\|v\|_{H^{1}\left(\Omega ; L_{M}^{2}(D)\right)}^{2},
\end{aligned}
$$

which shows that $H_{M}^{1,0}(\Omega \times D)$ and $H^{1}\left(\Omega ; L_{M}^{2}(D)\right)$ are isometrically isomorphic.

\section{A.3. Step 3: Compact embedding of $H_{M}^{1}(\Omega \times D)$ into $L_{M}^{2}(\Omega \times D)$}

We use the results of Step 2 to identify the space $L_{M}^{2}(\Omega \times D)$ with $L^{2}\left(\Omega ; L_{M}^{2}(D)\right)$ and the space $H_{M}^{1}(\Omega \times D)=H_{M}^{1,0}(\Omega \times D) \cap H_{M}^{0,1}(\Omega \times D)$ with $H^{1}\left(\Omega ; L_{M}^{2}(D)\right) \cap$ $L^{2}\left(\Omega ; H_{M}^{1}(D)\right)$.

Upon doing so, the compact embedding of $H_{M}^{1}(\Omega \times D)$ into $L_{M}^{2}(\Omega \times D)$ directly follows from the compact embedding of $H^{1}\left(\Omega ; L_{M}^{2}(D)\right) \cap L^{2}\left(\Omega ; H_{M}^{1}(D)\right)$ into $L^{2}\left(\Omega ; L_{M}^{2}(D)\right)$, implied by Theorem 2 on p.1499 in the paper of Shakhmurov ${ }^{33}$.

\section{References}

1. L. Ambrosio, Transport equation and Cauchy problem for BV vector fields, Invent. Math., 158 (2004), pp. 227-260.

2. F. AnToci, Some necessary and some sufficient conditions for the compactness of the embedding of weighted Sobolev spaces, Ricerche Mat., 52 (2003), pp. 55-71.

3. A. Arnold, P. Markowich, G. Toschni, And A. Unterreiter, On convex Sobolev inequalities and the rate of convergence to equilibrium for Fokker-Planck equations. Comm. PDE, 26 (2001), pp. 43-100.

4. J. W. BARrett, Ch. Schwab, AND E. SüLI, Existence of global weak solutions for some polymeric flow models, Math. Models Methods Appl. Sci., 15 (2005), pp. 939983.

5. J. W. BARRETT AND E. SÜLI, Existence of global weak solutions to some regularized kinetic models of dilute polymers, SIAM Multiscale Modelling and Simulation, 6 (2007) pp. 506-546.

6. J. W. BARRETT AND E. SÜLI, Numerical approximation of corotational dumbbell models for dilute polymers, IMA J. Numer. Anal. (submitted for publication).

7. J. W. BARRETT AND E. SÜLI, Finite element approximation of kinetic dilute polymer models with microscopic cut-off, (in preparation).

8. A. V. Bhave, R. C. Armstrong, and R. A. Brown, Kinetic theory and rheology of dilute, nonhomogeneous polymer solutions, J. Chem. Phys., 95 (1991), pp. 2988-3000. 
9. R. Bird, C. Curtiss, R. Armstrong, and O. Hassager, Dynamics of Polymeric Liquids, Volume 2: Kinetic Theory, John Wiley and Sons, New York, 1987.

10. P. Constantin, Nonlinear Fokker-Planck Navier-Stokes systems, Commun. Math. Sci., 3 (2005), pp. 531-544.

11. L. Desvillettes And C. Villani, On the trend to global equilibrium in spatially inhomogeneous entropy-dissipating systems: the linear Fokker-Planck equation, Comm. Pure Appl. Math., 54 (2001), pp. 1-42.

12. R. J. DiPerna And P.-L. Lions, Ordinary differential equations, transport theory and Sobolev spaces, Invent. Math., 98 (1989), pp. 511-547.

13. Q. Du, C. LIU, AND P. YU, FENE Dumbbell model and its several linear and nonlinear closure approximations, Multiscale Model. Simul., 4 (2005), pp. 709-731.

14. W. E, T. J. LI, AND P.-W. ZhANG, Well-posedness for the dumbbell model of polymeric fluids, Comm. Math. Phys., 248 (2004), pp. 409-427.

15. A. W. El-Kareh And L. G. Leal, Existence of solutions for all Deborah numbers for a non-Newtonian model modified to include diffusion, J. Non-Newtonian Fluid Mech., 33 (1989), pp. 257-287.

16. C. Foins, D. D. Holm, And E. S. Titi, The Navier-Stokes- $\alpha$ model of fluid turbulence, Phys. D, 152/153 (2001), pp. 505-519.

17. B. Jourdain, T. Lelièvre, AND C. LE BRIS, Numerical analysis of micro-macro simulations of polymeric fluid flows: A simple case, Math. Models Methods Appl. Sci., 12 (2002), pp. 1205-1243.

18. B. Jourdain, T. Lelièvre, And C. Le Bris, Existence of solution for a micro-macro model of polymeric fluid: The FENE model, J. Funct. Anal., 209 (2004), pp. 162-193.

19. B. Jourdain, T. Lelièvre, C. Le Bris, ANd F. Otto, Long-time asymptotics of a multiscle model for polymeric fluid flows, Arch. Rat. Mech. Anal., 181 (2006), pp. 97148.

20. R. Keunings, A survey of computational rheology, in Proceedings of the XIIIth International Congress on Rheology, Vol. 1, British Society of Rheology, Cambridge, UK, 2000, pp. 7-14.

21. A. Kufner, Weighted Sobolev Spaces, Teubner-Texte zur Mathematik, Teubner, 1980.

22. T. Li, H. Zhang, And P.-W. Zhang, Local existence for the dumbbell model of polymeric fluids, Comm. Partial Differential Equations, 29 (2004), pp. 903-923.

23. F.-H. Lin, C. LiU, AND P. ZHANG, On a micro-macro model for polymeric fluids near equilibrium, Comm. Pure Appl. Math., 60 (2007), pp. 838-866.

24. P.-L. LiOns AND N. MASMOUdi, Global solutions for some Oldroyd models of nonNewtonian flows, Chinese Ann. Math. Ser. B, 21 (2000), pp. 131-146.

25. P.-L. Lions AND N. Masmoudi, Global existence of weak solutions to some micromacro models, C. R. Math. Acad. Sci. Paris, 345 (2007), pp. 15-20.

26. A. Lozinski, C. Chauvière, J. Fang, And R. G. Owens, Fokker-Planck simulations of fast flows of melts and concentrated polymer solutions in complex geometries, J. Rheol., 47 (2003), pp. 535-561.

27. A. Lozinski, R. G. Owens, And J. FAng, A Fokker-Planck-based numerical method for modelling non-homogeneous flows of dilute polymeric solutions, J. Non-Newtonian Fluid Mech., 122 (2004), pp. 273-286.

28. N. Masmoud, Well posedness of the FENE dumbbell model of polymeric flows, Preprint, 2007. http://www.math.nyu.edu/faculty/masmoudi/index.html

29. H.-C. Öttinger, Stochastic Processes in Polymeric Fluids, Springer-Verlag, Berlin, 1996.

30. F. Отto and A. Tzavaras, Continuity of Velocity Gradients in Suspensions of RodLike Molecules, SFB Preprint 147, Institute of Applied Mathematics, University of 
January 15, 2008 18:6 WSPC/INSTRUCTION FILE barrett-suli-m3assubmission.final

Bonn, Bonn, Germany, 2004.

31. M. RENARDY, An existence theorem for model equations resulting from kinetic theories of polymer solutions, SIAM J. Math. Anal., 22 (1991), pp. 313-327.

32. J. D. SChIEBER, Generalized Brownian configuration field for Fokker-Planck equations including center-of-mass diffusion, J. Non-Newtonian Fluid Mech., 135 (2006), pp. 179-181.

33. V. B. Shakhmurov, Embedding and maximal regular differential operators in Sobolev-Lions spaces, Acta Math. Sin. (Engl. Ser.), 22 (2006), pp. 1493-1508.

34. J. Simon, Compact sets in the space $L^{p}(0, T ; B)$ ' Ann. Math. Pura. Appl., 146 (1987), pp. 65-96.

35. R. Temam, Navier-Stokes Equations. Theory and Numerical Analysis, 3rd ed., Stud. Math. Appl. 2, North-Holland, Amsterdam, 1984.

36. H. Triebel, Interpolation Theory, Function Spaces, Differential Operators, 2nd ed., Johann Ambrosius Barth, Heidelberg, 1995.

37. P. YU, Q. DU, AND C. LiU, From micro to macro dynamics via a new closure approximation to the FENE model of polymeric fluids, Multiscale Model. Simul., 3 (2005), pp. 895-917.

38. H. ZhANG AND P. ZhANG, Local existence for the FENE-dumbbell model of polymeric fluids, Arch. Ration. Mech. Anal., 181 (2006), pp. 373-400. 